\title{
ARTICLE
}

\section{In vivo imaging of clock gene expression in multiple tissues of freely moving mice}

\author{
Toshiyuki Hamada ${ }^{1}$, Kenneth Sutherland ${ }^{2}$, Masayori Ishikawa ${ }^{1,2,3,4}$, Naoki Miyamoto ${ }^{2}$, Sato Honma ${ }^{5}$, \\ Hiroki Shirato ${ }^{4,6} \&$ Ken-ichi Honma ${ }^{5}$
}

Clock genes are expressed throughout the body, although how they oscillate in unrestrained animals is not known. Here, we show an in vivo imaging technique that enables long-term simultaneous imaging of multiple tissues. We use dual-focal 3D tracking and signal-intensity calibration to follow gene expression in a target area. We measure circadian rhythms of clock genes in the olfactory bulb, right and left ears and cortices, and the skin. In addition, the kinetic relationship between gene expression and physiological responses to experimental cues is monitored. Under stable conditions gene expression is in phase in all tissues. In response to a long-duration light pulse, the olfactory bulb shifts faster than other tissues. In $\mathrm{Cry}^{-/-} \mathrm{Cry}^{-/-}$arrhythmic mice circadian oscillation is absent in all tissues. Thus, our system successfully tracks circadian rhythms in clock genes in multiple tissues in unrestrained mice.

\footnotetext{
${ }^{1}$ Department of Applied Molecular-Imaging Physics, Hokkaido University Graduate School of Medicine, Sapporo 060-8638, Japan. ${ }^{2}$ Department of Medical Physics and Engineering, Hokkaido University Graduate School of Medicine, Sapporo 060-8638, Japan. ${ }^{3}$ Department of Biomedical Science and Engineering, Hokkaido University Graduate School of Health Science, Sapporo 062-0812, Japan. ${ }^{4}$ Global Station for Quantum Medical Science and Engineering, Global Institution for Collaborative Research and Education (GI-CoRE), Hokkaido University, Sapporo 060-8638, Japan. ${ }^{5}$ Department of Chronomedicine, Hokkaido University Graduate School of Medicine, Sapporo 060-8638, Japan. ${ }^{6}$ Department of Radiation Medicine, Hokkaido University Graduate School of Medicine, Sapporo 060-8638, Japan. Correspondence and requests for materials should be addressed to M.I. (email: masayori@med.hokudai.ac.jp) or to K.H. (email: kenhonma2011@gmail.com).
} 
C ircadian rhythms in physiology and behaviour in mammals are regulated by a master clock located in the suprachiasmatic nucleus (SCN). Clock genes have critical roles in expressing cellular circadian rhythms, primarily based on a cell autonomous molecular feedback loop ${ }^{1}$. Clock gene expression is not limited to the SCN, with expression observed in a variety of tissues ${ }^{1-5}$. However, the expression pattern of clock genes outside the SCN is not well understood, partly owing to a lack of techniques for simultaneous monitoring of circadian clock gene rhythms in specific tissues, and to detect output functions in the absence of physical restraints.

The bioluminescent reporter enzyme firefly luciferase (luc) and its substrate D-luciferin (luciferin) have been used to generate optical imaging signals with high sensitivity in living animals ${ }^{6}$, and have been adapted successfully to whole-body imaging of clock genes in anaesthetized mice using a charge-coupled device (CCD) camera $^{7,8}$. However, anaesthesia is reported to alter the clock gene expression ${ }^{9-15}$. Saini et al. ${ }^{16}$ measured the rhythmic bmall expression only in the liver of a freely moving mouse with a CCD camera located outside the body.

Monitoring of clock gene expression in vivo has also been attempted using a bioluminescent reporter, either with an optical fibre in the $\mathrm{SCN}^{17}$, or using a photon detector located outside the body. In the latter case, bioluminescence emitted from tissuespecific reporters was collected efficiently using a conical wall that channels photons toward a photomultiplier tube (PMT), and the emitting tissue was identified using a tissue-specific reporter ${ }^{16}$. Although promising, these approaches have limitations. For example, monitoring clock gene expression using an implanted optical fibre only enables focusing on the target area in a limited space, and insertion of an optical fibre may damage the area surrounding the $\operatorname{target}^{18}$. In the case of bioluminescent detection using a PMT external to the body, tissue-specific bioluminescence may need to be quantified if the target is relatively large and is located in deep areas inside the body. In addition, three-dimensional (3D)-calibration of signal strength is necessary.

Here, we show a method to quantitatively monitor clock gene expression simultaneously in multiple regions of freely moving mice over long durations. The challenge was to calibrate the bioluminescence intensity in moving targets. To overcome the technical challenge of intensity changes with time-of-day and distance from the recording apparatus, we developed a dual-focal 3D tracing (DuFT) technology and a signal-intensity calibration technique (SICT) and combined these two systems into a software application for analysing gene expression, which we call 'Mouse Tracker'.

These techniques enable us to monitor the circadian rhythm of clock gene expression concurrently in multiple tissues with unrestrained mice. The results indicate that the olfactory bulb (OB) shifts faster than other tissues (right and left ears and cortices, and skin) in response to a long-duration light pulse.

\section{Results}

The in vivo imaging system used in this study consists of two novel techniques, DuFT and SICT (Fig. 1a,b and Supplementary Fig. 1a-g). Bioluminescent signals representing Per1 expression were produced following intraperitoneal administration of luciferin through an application system (Fig. $1 \mathrm{~b}$ and Supplementary Fig. 1d,e) into freely moving Per1-luc mice (Fig. 1c). The following sections describe the principles and detailed methods of each technology.

DuFT for detecting the 3D positions of scintillators. To serve as position markers, scintillators attached to the skin of the back (hereafter referred as skin) and the head were used to detect the regions of interest (ROIs), namely $\mathrm{OB}$, ears and cerebral cortices (hereafter referred as cortex; Fig. 1c). To detect the 3D positions of a scintillator in freely moving animals in the recording cage (Supplementary Fig. 1f), we developed tracking software based on stereo photography ${ }^{19-21}$ and pattern-matching 22 principles. Two cameras captured a pair of images (left; $\mathrm{L}(\mathrm{L} x, \mathrm{~L} y)$ and right $\mathrm{R}(\mathrm{R} x, \mathrm{R} y))$. From the $2 \mathrm{D}$ coordinates of the scintillators in each camera's image, two straight lines passing through a scintillator were calculated using transformation matrices $\left(M_{1}\right.$ and $M_{r}$; Fig. 1d) (see the 'Methods' section for details). The 3D coordinates of the scintillator $\left(\mathrm{S}\left(\mathrm{S}_{x}, \mathrm{~S}_{y}, \mathrm{~S}_{z}\right)\right)$ were determined by calculating the intersection of these two lines (Fig. 1d). In situations when the signal of the scintillator is lost due to the angle of the two EM-CCD cameras, the signal can be reacquired automatically when the mouse moves into a more appropriate position. Intensity of the LED-light-induced fluorescent signals (Supplementary Fig. 2a-c) from the scintillators was set (Supplementary Fig. 1g) to be roughly equivalent to the bioluminescent signals from the skin of the freely moving mice (Fig. 1c).

Signal-intensity calibration technique. We developed a number of algorithms to locate the scintillators using a model image for pattern matching to identify the associated ROIs. For the skin, scintillators were attached on a patch of shaved skin. The ROI was assumed to be at the centre of the baseline of the triangle formed by the three scintillators. The OB ROI was assumed to be at the centroid of the triangle formed by the three scintillators (Fig. 1f). For the head, an image of one of the three scintillators placed on the head was used as a model for pattern matching. To identify left and right ears/cortices (Supplementary Fig. 3), we further developed a method using a model head image for pattern matching (Fig. 2a,b). Adding the model head image for pattern matching resulted in decreased mismatching of the scintillator image. Selection of the model head image for pattern matching (model image in Supplementary Fig. 4 is for OB and model image in Fig. $2 \mathrm{~b}$ is for OB, left ear, right ear, left cortex, right cortex) is based on the number of target areas to be measured.

The intensity of the bioluminescent signal varies with the position of the animal within the recording cage due to variations in distance from the camera. To calibrate the bioluminescent signal obtained from individual ROIs, we calibrated signal intensity using the $3 \mathrm{D}$ coordinates of the target area on the mouse by referring to a calibration table (Supplementary Fig. 5a-f; see the 'Methods' section for details) which specifies the ratio of the calibration value at each point in the imaging space.

The bioluminescence from the target area was calculated from the mean ROI pixel intensity (mean pixel value; shown with a yellow circle in Fig. 1f) for each image from the EM-CCD cameras. In this way, Per1 expression in ROIs was automatically marked with circles (identification), traced (Supplementary Movie 1) and quantified (Supplementary Fig. 6).

The spatial resolution was estimated from the cage positioning and the pattern-matching errors. The maximum error in cage positioning was $0.21 \mathrm{~mm}$ which was calculated from 1,815 measurements of 605 different points (each 3 times) in the cylindrical matrix (r, $5 \mathrm{~cm}$; height, $4 \mathrm{~cm}$; Supplementary Fig. 5). We estimated the cage positioning error to be $0.63 \mathrm{~mm}$ which was 3 times of the largest standard deviation (s.d.) of the mean. The pattern-matching error was calculated to be $1.50 \mathrm{~mm}$ (3 s.d.), when matching of $>30 \%$ was accepted as valid. Thus, the spatial resolution with a confidence range of $99.9 \%$ is $1.63 \mathrm{~mm}$. 
a

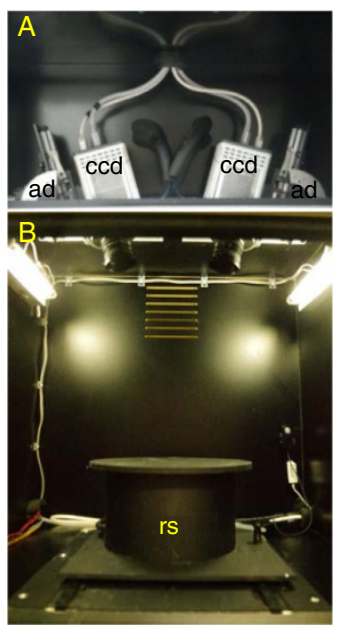

d

Left EM-CCD camera Right EM-CCD camera

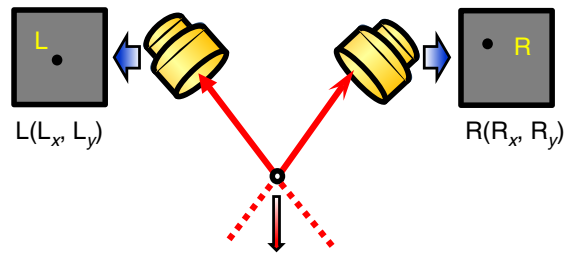

$\mathrm{S}\left(\mathrm{S}_{x}, \mathrm{~S}_{y}, \mathrm{~S}_{z}\right)$

Cross section of LS and RS straight lines

$\left[\mathrm{aM}_{\mathrm{l}}\right]=[\mathrm{b}] \quad\left[\mathrm{aM}_{\mathrm{r}}\right]=[\mathrm{b}]$

[a]=any position on the LS and RS straight lines

$[b]=2 \mathrm{D}$ target position; $\mathrm{L}\left(\mathrm{L}_{x}, \mathrm{~L}_{y}\right)$ or $\mathrm{R}\left(\mathrm{R}_{x}, \mathrm{R}_{y}\right)$

$M_{1}$ or $M_{r} ; 3 D$ transformation matrix b

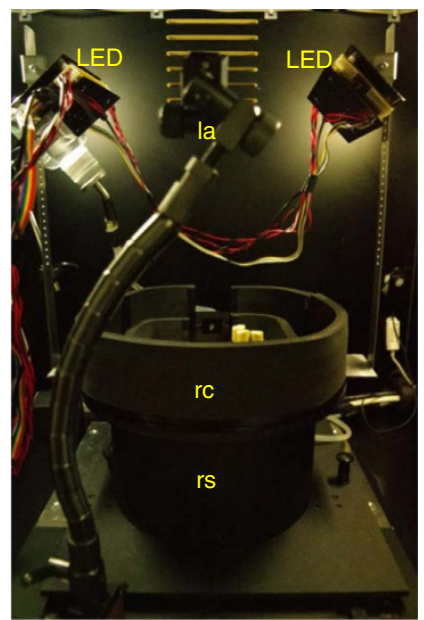

C

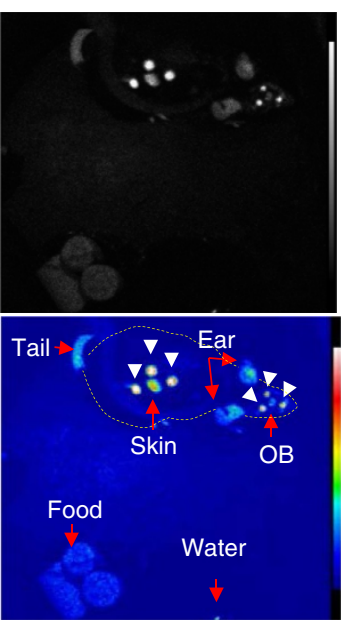

e $10 \min (0.5$ or $1.0 \mathrm{~s}$ exposure time)

Recording within each 0.5 or $1 \mathrm{~h}$ Mouse capture

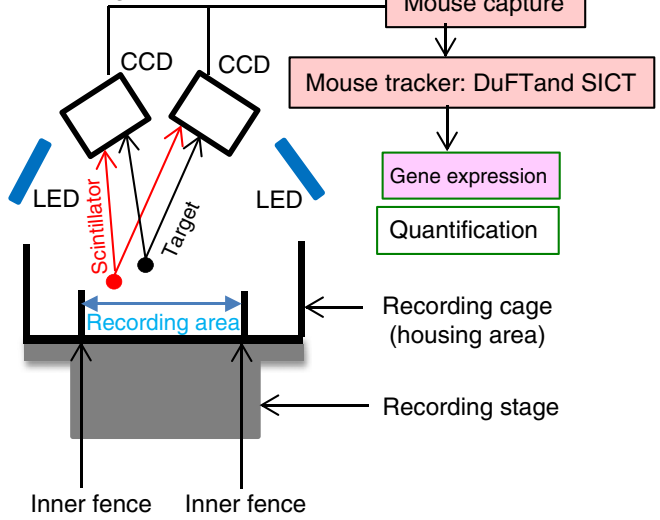

DuFT image

f

Raw image

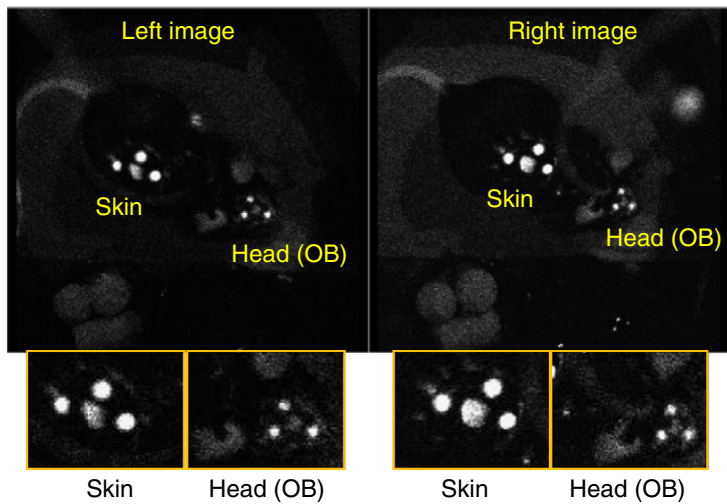

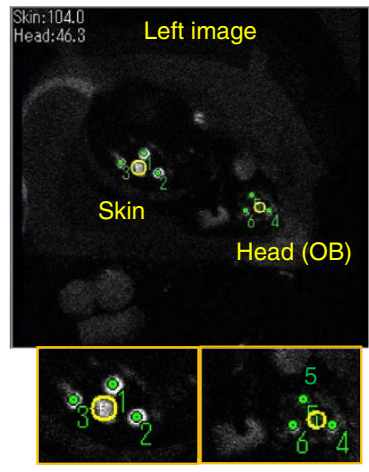

Skin

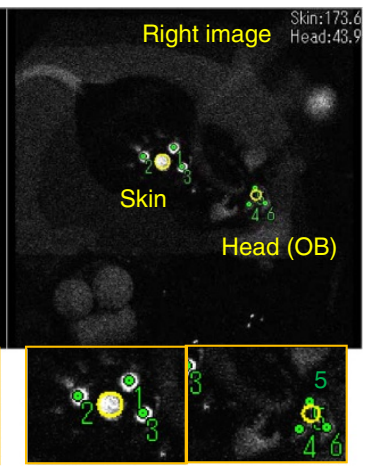

Skin

Head (OB)

Figure 1 | In vivo imaging system. (a) Inside the EM-CCD camera box (A) and imaging box (B). The upper photo shows two high-sensitive EM-CCD cameras (ccd) with an angle adjuster (ad). The lower photo shows two fluorescent lights and the recording stage ( $r$ ) in the ventilated imaging box. (b) DuFT recording system. Recording cage ( $r c$ ) on the recording stage, two LED lights and luciferin application system (la) are shown. (c) Per7-luc mouse with scintillators attached to the head and skin exposed to the LED light in freely moving condition. The fluorescence signal from the scintillators and bioluminescent signals from the target area are acquired by EM-CCD cameras in the raw data (upper) and pseudo-colour data (lower). The arrowhead shows three scintillators around the skin target and those around the OB targets. The dotted line shows the shape of the mouse body. Luciferin ( $40 \mathrm{mg} \mathrm{ml}^{-1}$ ) was supplied by an iPRECIO pump $\left(15 \mu \mathrm{l} \mathrm{h}^{-1}\right.$ ). Images were captured at $0.5 \mathrm{~s}$ per frame. (d) Determination of the 3D coordinates of a scintillator $\left\{\mathrm{S}\left(\mathrm{S}_{x}, \mathrm{~S}_{y}, \mathrm{~S}_{z}\right)\right\}$ in the recording cage. The 3D transformation matrix from the 2D coordinates ( $x$-axis limitation; $0<x<512, y$-axis limitation; $0<y<512)$ on a pair of images $\{$ left; $L(L x, L y)$ and right; $R(R x, R y)$ \} was used to make a straight line passing through points $L$ and $S$ or $R$ and $S$. The $3 D$ coordinates of the scintillator $\left\{\mathrm{S}\left(\mathrm{S}_{x}, \mathrm{~S}_{y}, \mathrm{~S}_{z}\right)\right\}$ are calculated by intersecting these two lines. (e) In vivo imaging system overview. Data are saved in the computer at each 0.5 or $1 \mathrm{~h}$. Saved data are analysed by 'Mouse Tracker' consisting of pattern match program, tracking program and 3D calibration program. Quantification analysis yields the gene expression levels. (f) Representative image of Per1 expression of freely moving Per7-luc transgenic mouse by two EM-CCD cameras. DuFT image is made by analysing the raw image with 'Mouse Tracker'. Three scintillators on the head and skin are identified by DuFT. Green spots in the image indicate the scintillator positions determined by the software, numbered by descending pattern-matching score. 
a

Model scintillator image for pattern matching

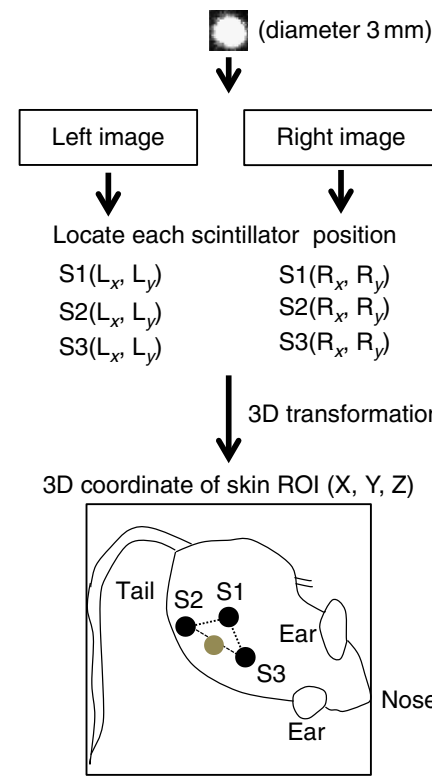

S1, S2, S3; scintillator (diameter $3 \mathrm{~mm}$ ) Skin ROI b

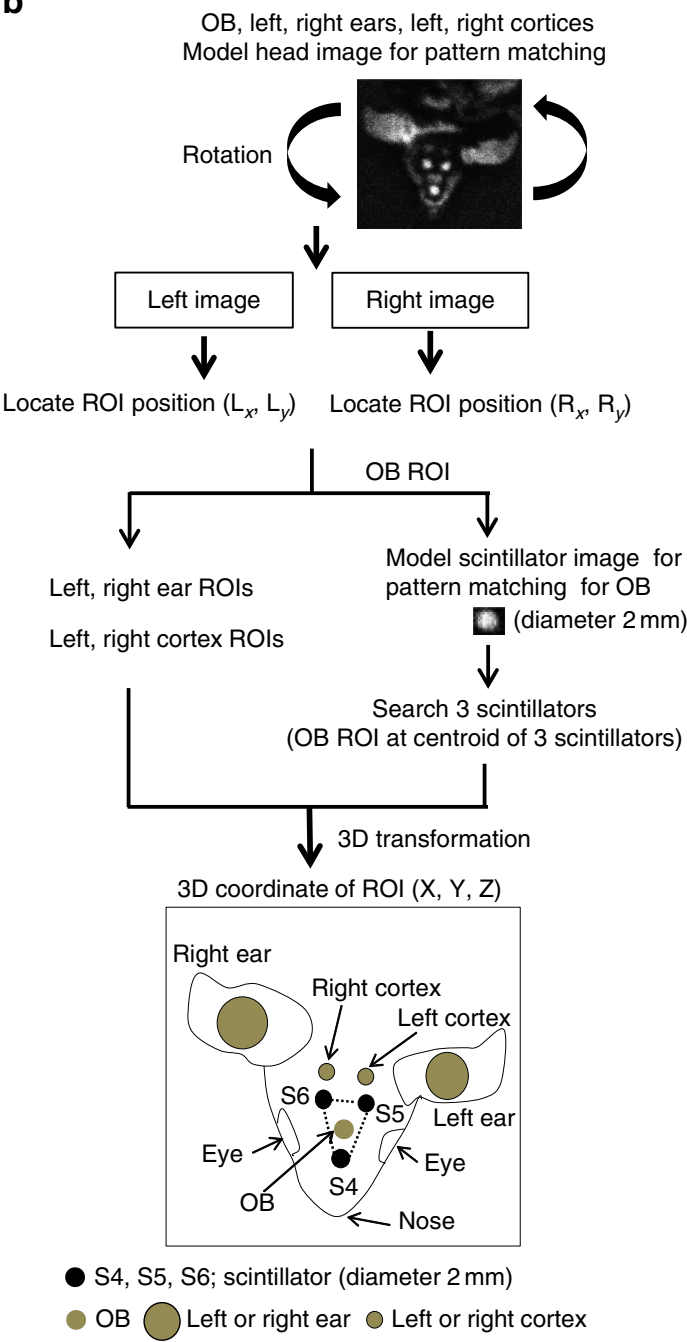

Figure 2 | Determination of ROI positions in six areas of freely moving mice. First, the frames with OB, skin, ear, cortex and three scintillators on each image are selected by 'Mouse Tracker'. (a) For skin, three scintillator ROls are identified using a model scintillator image. The skin ROI was assumed to be at the centre of the baseline of the triangle formed by the three scintillators. (b) For OB, ear and cortex ROls, a model head image is used as a search model for pattern matching in all frames of the video with any angle of rotated versions of the model. Ear and cortex ROls are defined relative to the top-left corner of the model head image. The OB ROI was assumed to be at the centroid of the triangle formed by the three scintillators. Next, 3D coordinates of ROI in each area are computed by transformation matrix (see the 'Methods' section for details). 3D coordinates of ROls are illustrated in the skin and head of the mouse.

Perl and Bmall expression in $\mathrm{OB}$ and skin of transgenic mice. Per1 and Bmall expression rhythms in vivo, which was compared with ex vivo expression in the $\mathrm{OB}$ and skin of freely moving mice under constant darkness (DD) are shown in Fig. 3a,b. In the OB and skin, circadian rhythms in Per1 gene expression were robust, exhibiting peak expression in the subjective evening as previously reported $^{23,24}$. In contrast, Bmal1 showed circadian gene expression that was antiphasic to that of Per1. The OB results were consistent with ex vivo experiments (Fig. 3c) in which Per1 and Bmall expression showed a similar antiphasic relationship in horizontal brain slices of the whole OB $(0.3 \mathrm{~mm}$ slice thickness). Circadian peak and trough of Per1-luc expression rhythm on the third culture day were observed at 18:12 $\pm 0: 36$ and 5:12 \pm 0:48 local time (mean \pm s.d., $n=3$, period $=23.4 \pm 0.3 \mathrm{~h}$ ), respectively. Whereas, the peak and trough of Bmal1-ELuc expression rhythm on the fourth culture day were observed at 5:00 $\pm 1: 30$ and 15:15 \pm 0:54 (mean \pm s.d., $n=3$, period $=22.6 \pm 0.3 \mathrm{~h}$ ). These results were consistent with our previous in vivo study of mRNA expression ${ }^{23}$.
Supplementary Movies 2 and 3 show Per 1 gene expression at 19:00 hours and 07:00 hours, respectively on the first day in DD. The recorded target areas were OB and skin. At 19:00 hours the mouse was eating food and moving around in the cage. Representative images at each time point are shown in Supplementary Fig. 7a. The 3D plots tracking the OB show behaviour in the cage at each time point (Supplementary Fig. 7b). At 07:00 hours in the morning, the mouse was inactive and remained in one position (Supplementary Fig. 7b). Calibrated bioluminescent intensity of Per1-luc expression in the $\mathrm{OB}$ and skin of freely moving mice in the recording cage were confirmed by extracting the data in which the mouse was stationary during a 10 -s period (changes in spatial position of $\leq 1 \mathrm{~mm}$; Supplementary Fig. 8).

Continuous luciferin administration. To quantify the bioluminescent signal at the whole-body level for an extended period of time, the luciferin concentration in blood should be stable over 
a
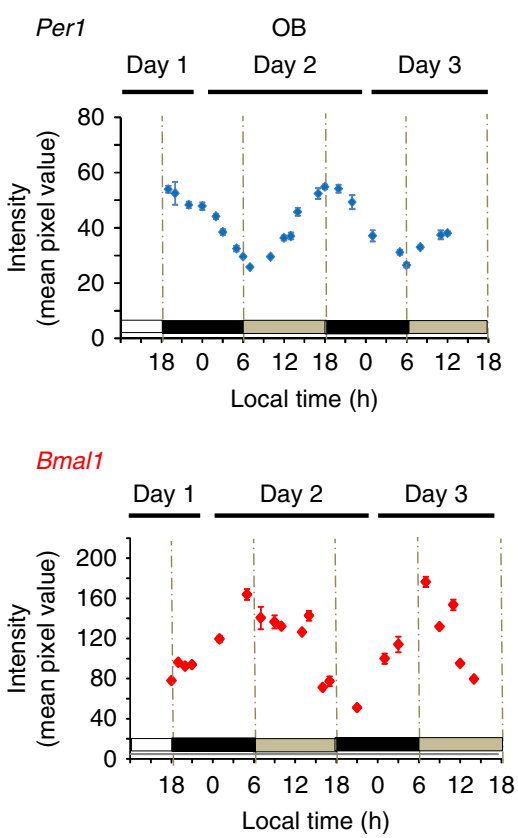

b
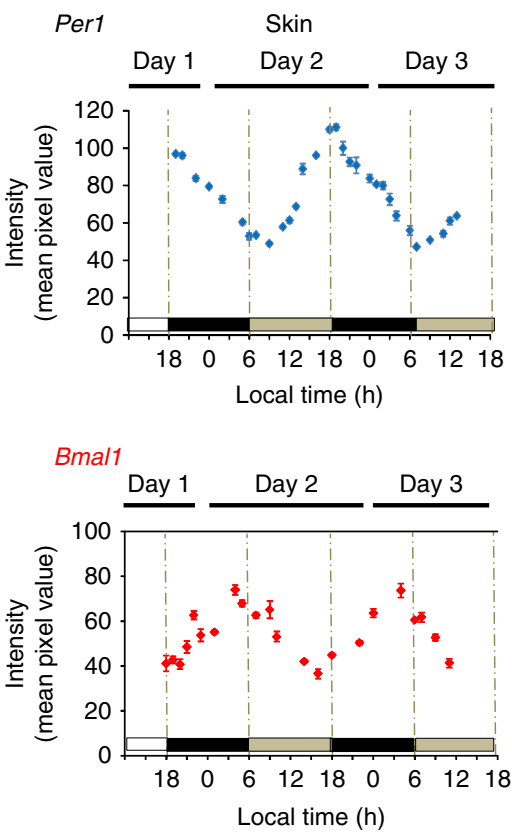

c
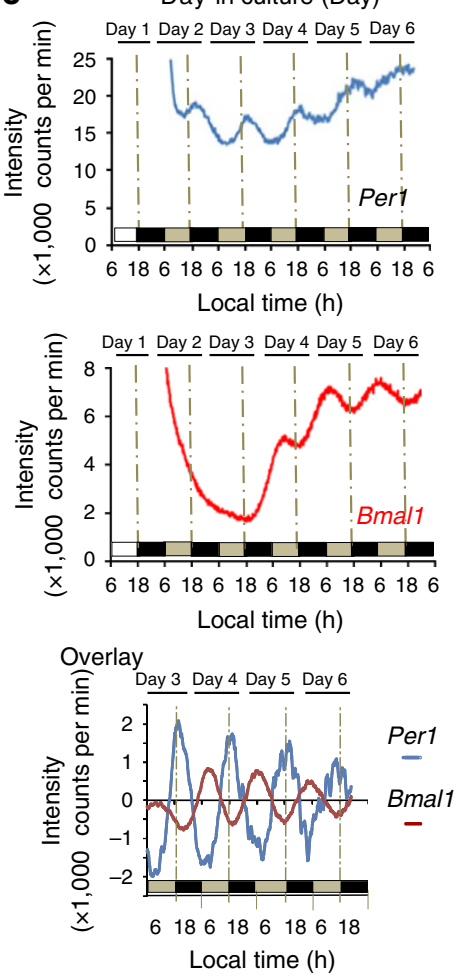

Day in culture (Day) d

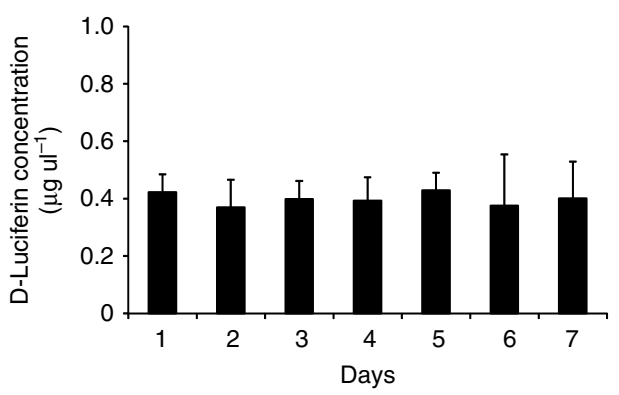

e

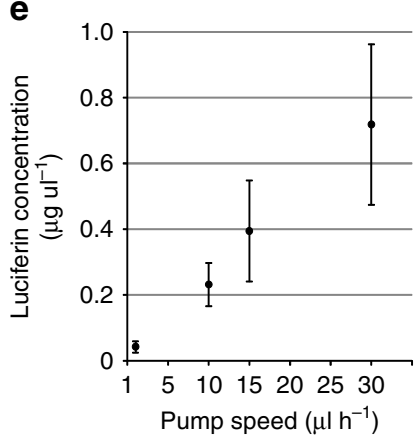

f
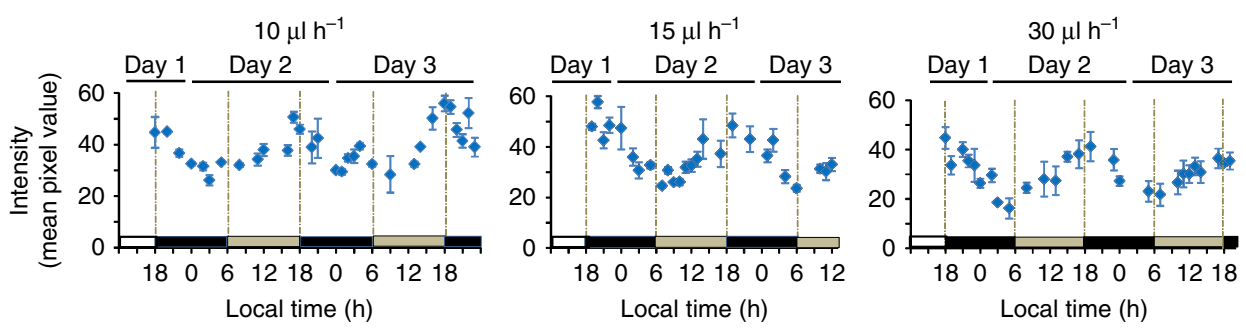

Figure 3 | Circadian Per1 and Bmal1 expression rhythm of the transgenic mice. In vivo imaging of Per1 (a) and Bmal1 (b) expression from OB and skin in freely moving mice. Luciferin was supplied by an iPRECIO pump $\left(15 \mu \mathrm{lh}{ }^{-1}\right.$ for Per1-luc and $30 \mu \mathrm{lh}^{-1}$ for Bmal7-enhanced beetle luciferase (ELuc)). Images were captured for $10 \mathrm{~min}$, at $0.5 \mathrm{~s}$ per frame for Per-luc and $1.0 \mathrm{~s}$ per frame for Bmal1-ELuc, at 1-h intervals. (c) Ex vivo imaging of Per1 and Bmal1 expression from the OB. Per1-luc and Bmal1-ELuc activities in the horizontal whole bilateral OB slices recorded for 1 min at every 10 min intervals for 6 days. The abscissa indicates days in culture. Day 0 is the day of slice preparation. The baseline-corrected bioluminescence was computed by subtracting the $24-\mathrm{h}$ moving average from the original data. (d) Luciferin concentration (mean \pm s.d.) in the plasma for 7 days. Luciferin concentration in plasma was stable for 7 days when luciferin $\left(40 \mathrm{mg} \mathrm{ml}^{-1}\right.$ ) was supplied at a perfusion rate of $15 \mu \mathrm{h} \mathrm{h}^{-1}$ using an iPRECIO pump. (e) The relationship between luciferin concentration (mean \pm s.d.) and perfusion rate. (f) Rhythmic Per1 expression of the transgenic mice in vivo at various perfusion rates $\left(40 \mathrm{mg} \mathrm{ml}^{-1}\right)$. Per1-luc mice implanted with an osmotic pump $\left(1 \mu \mathrm{h} \mathrm{h}^{-1}\right)$ into the peritoneal cavity exhibited low plasma luciferin concentration of $0.04 \pm 0.02 \mu \mathrm{g} \mu \mathrm{l}^{-1}$, and did not show detectable bioluminescent signals.

the recording period. In the present study, the plasma luciferin concentration was maintained at a stable level for 7 days (Fig. 3d). By maintaining the plasma luciferin concentration over $0.2 \mu \mathrm{g}^{-1}$, we successfully detected rhythmic bioluminescent emission in freely moving mice (Fig. 3e,f and Supplementary
Fig. 9a-d). Using an osmotic or iPRECIO pump, Per1-luc mice were administered luciferin at $1,10,15$ or $30 \mu \mathrm{hr}^{-1}$, and the plasma luciferin concentration in each mouse was $0.04 \pm 0.02$, $0.23 \pm 0.07, \quad 0.39 \pm 0.15$ and $0.72 \pm 0.24 \mu \mathrm{g} \mu \mathrm{l}^{-1}$, respectively (Fig. 3e). 
Plasma luciferin concentrations increased depending on the speed of dosage administration, and we were able to obtain images of Per1 ( $0.5 \mathrm{~s}$ EM-CCD camera exposure time) and monitor circadian Per1 expression in freely moving mice at plasma luciferin levels above $0.2 \mu \mathrm{g} \mu \mathrm{l}^{-1}$ (Fig. 3f). Under these conditions, plasma luciferin concentrations were stable over a $24-\mathrm{h}$ period. Therefore, we chose conditions where plasma luciferin concentrations exceeded $0.2 \mu \mathrm{g} \mu \mathrm{l}^{-1}$.

Per1-luc expression in freely moving wild-type mice. Per1-luc expression in the OB, skin, left ear, right ear, left cortex and right cortex was measured continuously for several days in freely moving mice under $\mathrm{DD}^{23-25}$ (Fig. 4a,b and Supplementary Movie 4). Circadian rhythms in Perl gene expression were detected in all ROIs examined with a peak during the early night and a trough during the early morning (Fig. 4c). In all Per1-luc mice examined, the six ROIs showed similar circadian phases in Per1 expression. Circadian rhythmicity of quantified Per1 expression analysed by the Cosinor program ${ }^{26}$ (see the 'Methods' section for details) is shown in Supplementary Tables 1 and 2.

Per1-luc expression in freely moving Cry $1^{-/-}$Cry2 $2^{-/-}$mice. We examined Per1-luc in Cryptochrome 1 (Cry1) and Cryptochrome 2 (Cry2) double-knockout (Cry1-/-/Cry2 $2^{-/-}$-Per1luc) mice. Cry 1 and Cry2 are components of a circadian feedback loop that generates circadian oscillation ${ }^{27}$. Adult $C r y 1^{-/-} /$ Cry $2^{-1-}$-Per1-luc mice are known to be aperiodic in behaviour and clock gene expression in the SCN under DD. As demonstrated in Fig. 5a-c and Supplementary Movie 5, Cry $1^{-1-}$ / Cry $2^{-/-}$-Per1-luc mice did not exhibit a significant circadian rhythm in Per1 expression with higher expression levels observed throughout the day, compared with the wild-type mice, in all ROIs examined (Supplementary Table 3 ). This result excludes the possibility that the observed $24-\mathrm{h}$ rhythm is an artefact.

Effects of a light pulse on circadian Per1-luc rhythms. The effects of a single light pulse on circadian Perl-luc expression rhythms were examined in all 6 ROIs. The mice were exposed to a light pulse between circadian time (CT) 12 and CT 20, where CT 12 was defined as the time of onset of activity (Supplementary Fig. 10). An 8-h light pulse produced a large and immediate phase-shift in locomotor activity rhythm $(-5.0 \pm 0.3 \mathrm{~h}, n=8$; Fig. $6 a)^{28}$. The phase-shift was almost complete on the day following the light pulse (1.6 \pm 0.7 days). The circadian Per 1 rhythms in the 6 ROIs were also phase-delayed but the degree of phase-shift was different among the ROIs. The circadian peak in the OB shifted the day following the light pulse $(-5.0 \pm 0.5 \mathrm{~h}$, $n=3$, Fig. $6 \mathrm{~b}, \mathrm{c})$ and did not shift further on the following days. Conversely, the circadian peaks in the other ROIs did not shift at all on the day following the light pulse. Phase-delay shifts were detected on the second day after the light pulse with a small secondary peak between the first and second circadian peaks after the light pulse observed in all 5 ROIs (skin, $5.8 \pm 0.7 \mathrm{~h}$; left ear, $6.0 \pm 1.0 \mathrm{~h}$; right ear, $5.0 \pm 1.0 \mathrm{~h}$; left cortex, $5.5 \pm 0.5 \mathrm{~h}$; right cortex, $6.0 \pm 1.0$ h, $n=3$; Fig. $6 \mathrm{~b}, \mathrm{c}$ and Supplementary Fig. 11). Thus, the response to a single 8 -h light pulse differed in the OB versus the other ROIs (skin, ears and cortices).

\section{Discussion}

The present study describes a new technology that enables the simultaneous, continuous, real-time monitoring in 3D space of gene expression in multiple sites over a period of several days in freely moving animals. Recently, tracking techniques for small, unmarked animals using pattern-matching techniques with one camera have been used for behavioural analysis in 2 dimensions ${ }^{29,30}$. Applying pattern-matching techniques to $3 \mathrm{D}$ imaging in the present study enabled the successful identification and tracking of the focal point of the imaging space in the recording cage. Tracking the position of markers on an animal in $3 \mathrm{D}$ enabled the identification of the direction of movement of the targets, and the simultaneous quantification of gene expression in multiple areas over a long duration. The spatial resolution of the present system is $1.63 \mathrm{~mm}$, meaning that two distinct spots $>1.63 \mathrm{~mm}$ apart are discriminated. The influence of lights scattering from an adjacent target area or from each of the triangle spots is negligible, as demonstrated by a dark area clearly demarcating the two target points. The influence of scattered light has been examined ${ }^{31}$. The light intensity diminishes sharply with distance from an illuminating spot to background levels in several pixels $\left(20-30 \mu \mathrm{m}^{3}\right)$.

The observed bioluminescent intensity changes depend on the time-of-day, the position of the target in the $3 \mathrm{D}$ space of the observation chamber and the angle of the CCD camera relative to the ROI. The DuFT method identifies the 3D position of a target based on the principles of stereo photography and the SICT algorithm calibrates the signal intensity in reference to a calibration table. These techniques enabled us to monitor the circadian rhythms of clock gene expression in multiple areas of an animal's body and their responses to intrinsic and extrinsic stimuli in unconstrained mice. The bioluminescence of the Per1 reporter used in our system was sufficient for detection with an EM-CCD camera using only a 0.5 -s exposure, confirming that it is possible to perform real-time analysis of the relationship between gene expression and behaviour. In the present study, only the areas on or near the body surface were selected as targets to monitor. In the future, utilization of strong tissue-specific reporters may enable the monitoring of multiple targets inside the body regardless of their location.

Previously, in vivo monitoring of clock gene expression in freely moving animals has been challenging ${ }^{16,17}$. The advantage of the present method over previous studies is threefold. First is the capability of quantifying the gene expression with $3 \mathrm{D}$ calibration, which was lacking in the previous studies. Second is the multi-region monitoring capability, enabling the analysis of relationships among tissues or cell-specific changes/responses in gene expression, as was done here (Figs $4 b, c, 5 b, c$ and $6 b, c)$. Third advantage is the capability to quantitatively monitor behaviour in $3 \mathrm{D}$ space, which enables the analysis of gene expression in correlation with specific behaviours, such as drinking and eating, with the gene expression in a particular location. Thus, the present in vivo monitoring system represents a novel analytical tool for examining the relation between circadian rhythms in clock gene expression and physiological outputs.

The primary limitation of the present system is the luciferin delivery system, which limits the recording duration. Luciferin delivery requires connecting animals to a delivery pump (iPRECIO) with a feed line or implanting an osmotic pump. Furthermore, to estimate the transcription rate from bioluminescent intensity, the intracellular luciferin concentration needs to be sufficient to support the luciferin-luciferase reaction. We delivered luciferin at a rate that produced a constant plasma concentration $>0.2 \mu \mathrm{g} \mu \mathrm{l}^{-1}$. However, further improvements in the substrate delivery systems would facilitate studies of this kind.

Using the present system, we observed robust circadian rhythmicity in Per1 expression at six different areas on the body of freely moving mice. The circadian rhythm was not an artefact, as the system confirmed aperiodism of clock gene expression in adult Cry $1^{-1-} / C r y 2^{-I-}-$ Per1-luc mice. Interestingly, these six areas measured showed similar circadian peaks in Per1 expression 
a

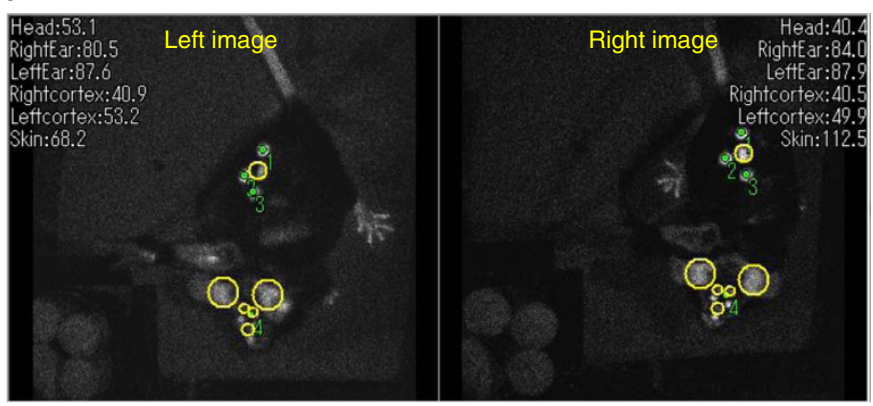

b
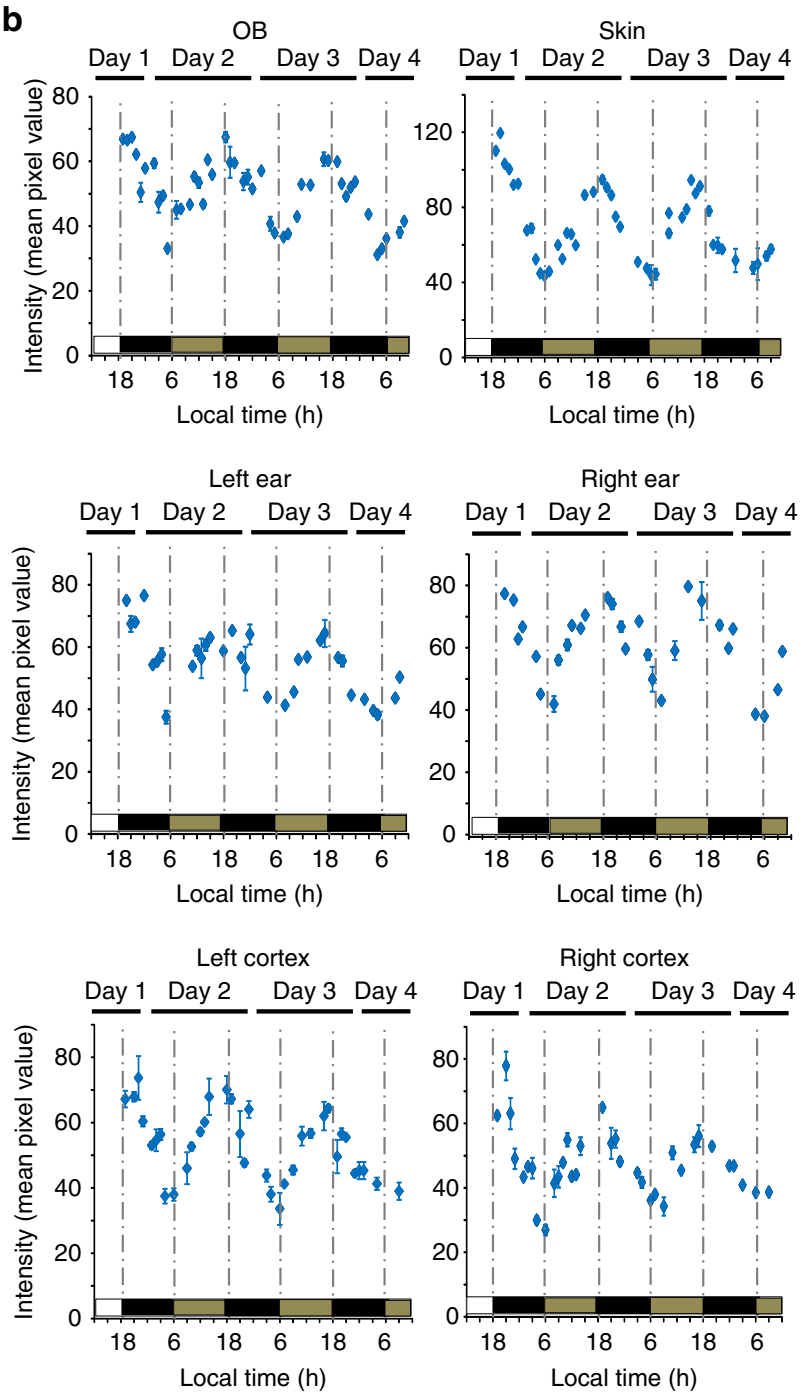

c $\mathrm{OB}$
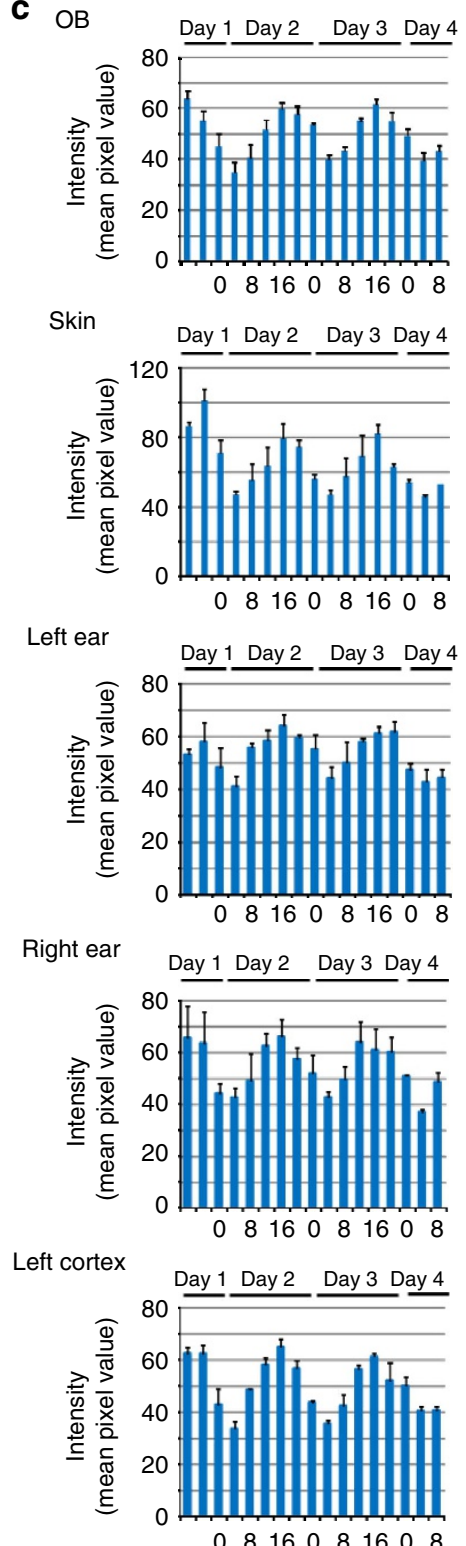

Right cortex

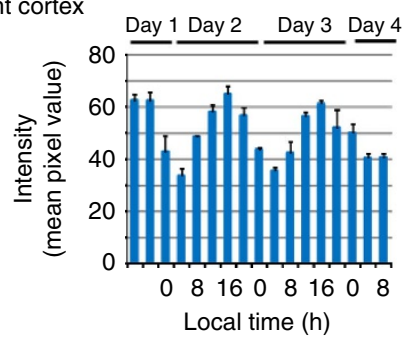

Figure 4 | In vivo imaging of Per1 expression in six areas of Per1-luc transgenic mice. Target areas (OB, skin, left ear, right ear, left cortex and right cortex) of Per7-luc (a) are automatically identified and traced. Yellow circles indicate identified areas by both EM-CCD cameras. Tracking marks and raw intensity within yellow circle areas are displayed in real time in the videos (30\% correlation). To quantify the gene expression in the ROI, mismatched frames were discarded during post-processing (see the 'Methods' section for details). Green spots in the image indicate the scintillator positions determined and numbered by the software in descending order of pattern matching score. Green spots in the image indicate the scintillator positions determined and numbered by the software in descending order of pattern matching score. (b) Representative Per1 expression rhythm in 6 areas of Per7-luc mice. Luciferin was supplied by iPRECIO pump ( $30 \mu \mathrm{l} \mathrm{hr}{ }^{-1}$ for animals). (c) Per1 expression in six areas of Per1-luc transgenic mice. The mean bioluminescence ( 4 h mean \pm s.e.m.) in six areas $(n=3 \sim 4$ animals). Per1 expression in six areas exhibited robust circadian rhythm. 
a

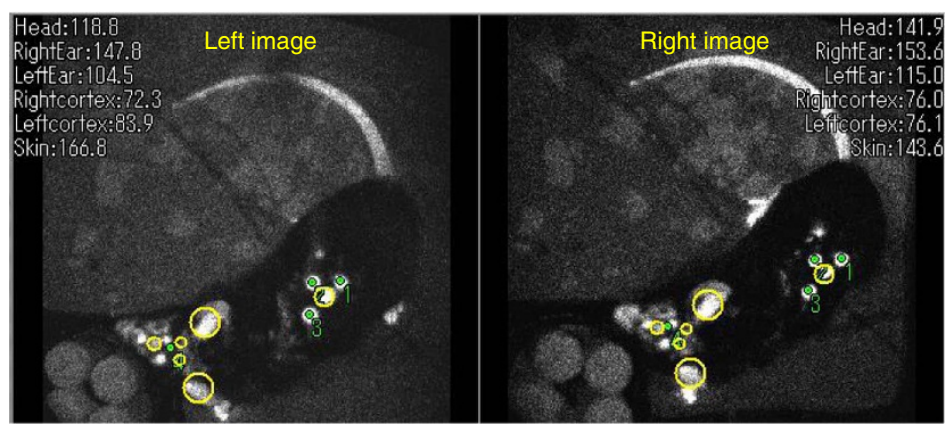

b

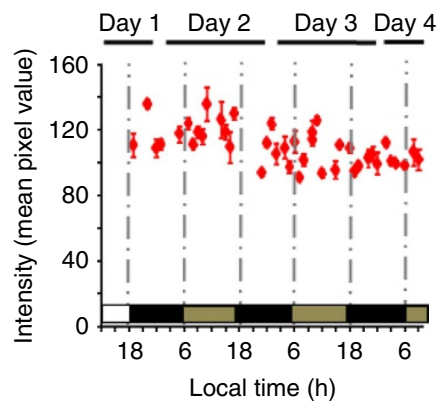

Left ear

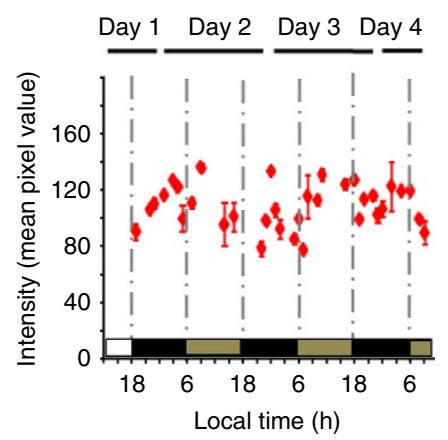

Left cortex

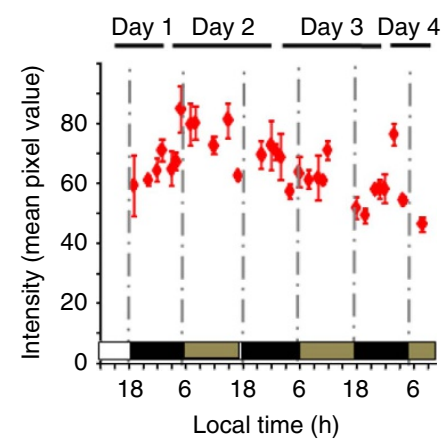

Skin

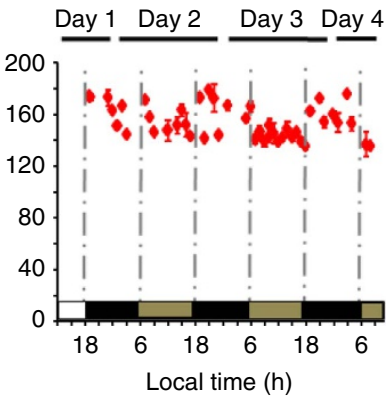

Right ear

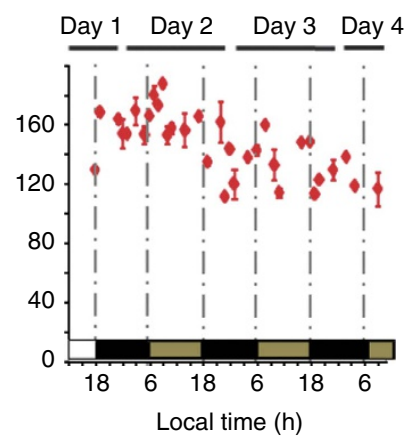

Right cortex

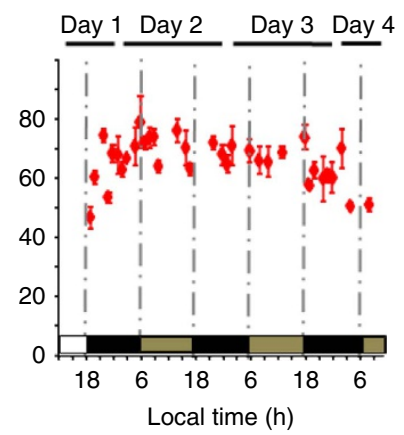

C

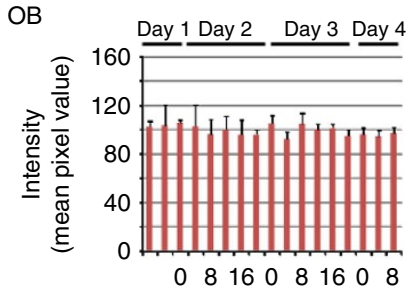

Skin

Left ear Day 1 Day 2 Day 3 Day 4

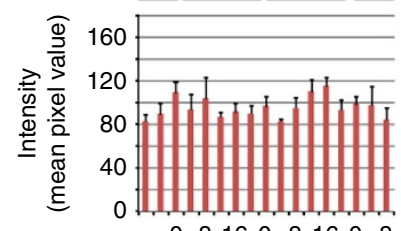

0816081608

Right ear Day 1 Day 2 Day 3 Day 4

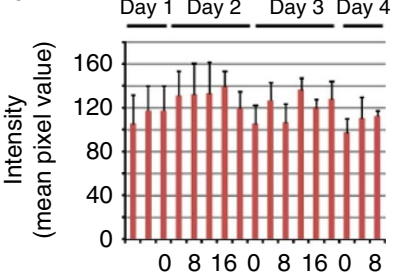

Left cortex

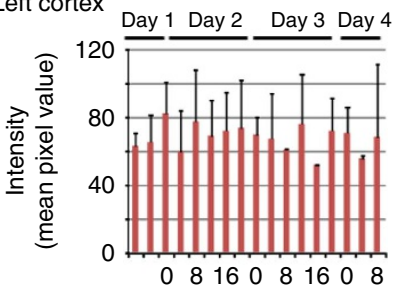

Right cortex

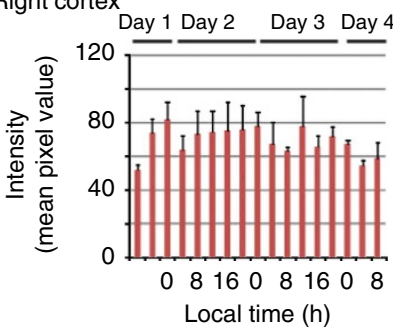

Figure 5 I In vivo imaging of Per1 expression in six areas of $\mathbf{C r y 1}^{-/}-/ \mathbf{C r y 2}^{-/}$- Per1-luc mice. (a) Per1 expression in the six different areas of the $\mathrm{Cry}^{-/-} / \mathrm{Cry}^{-/-}$-Per1-luc mice. Target areas (OB, skin, left ear, right ear, left cortex and right cortex) of Cry $1^{-/-} / \mathrm{Cry}^{-/-}-$Per1-luc mice were automatically identified and traced. Yellow circles indicate identified areas by both EM-CCD cameras. Tracking marks and raw intensity within yellow circle areas are displayed in real time in the videos (30\% correlation). To quantify the gene expression in the ROI, mismatched frames were discarded during post-processing (see the 'Methods' section for details). Green spots in the image indicate the scintillator positions determined and numbered by the software in descending order of pattern matching score. Green spots in the image indicate the scintillator positions determined and numbered by the software in descending order of pattern matching score. (b) Representative Per1 expression rhythm in six areas of $\mathrm{Cry} 1^{-/-} / \mathrm{Cry}^{-/-}-\mathrm{Per} 7-\mathrm{luc}$ mice. Luciferin $\left(40 \mathrm{mg} \mathrm{ml}^{-1}\right)$ was applied into the intraperitoneal cavity by iPRECIO pump (30 $\mu \mathrm{l} \mathrm{hr}^{-1}$ for animals). (c) Per1 expression in six areas of $\mathrm{Cry}^{-/-} / \mathrm{Cry}^{-/-}$-Per1-luc transgenic mice. The mean bioluminescence (4 hr mean \pm s.e.m.) in six areas $(n=3 \sim 4$ animals). Per1 expressions in six areas show no circadian rhythm. 
a

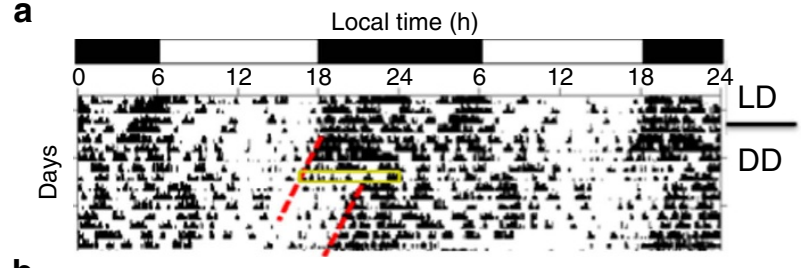

b

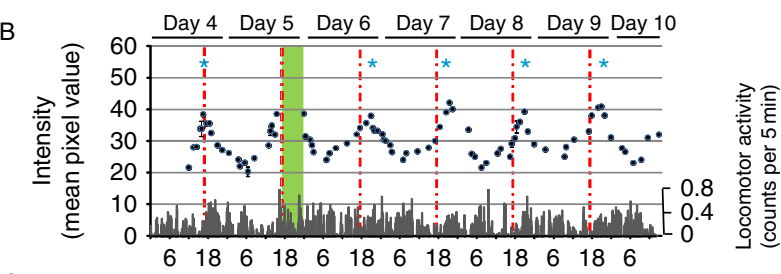

Skin

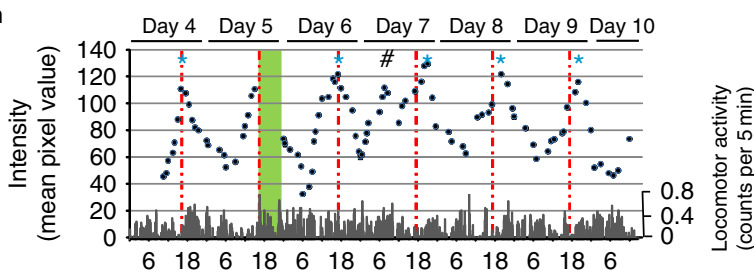

Left ear

$\begin{array}{lllllllllllll}6 & 18 & 6 & 18 & 6 & 18 & 6 & 18 & 6 & 18 & 6 & 18 & 6\end{array}$

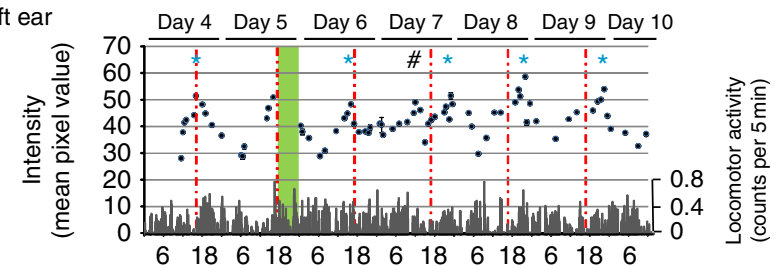

Right ea

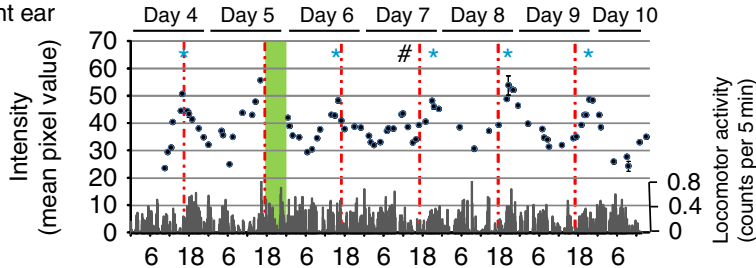

Left cortex

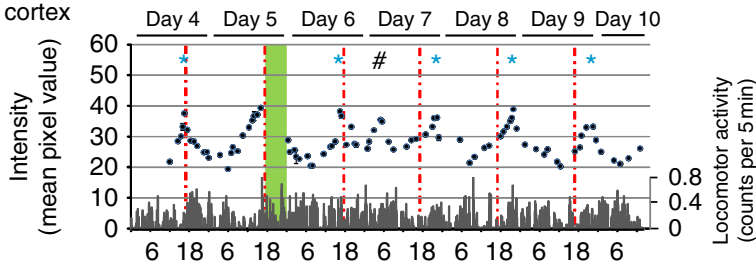

Right cortex

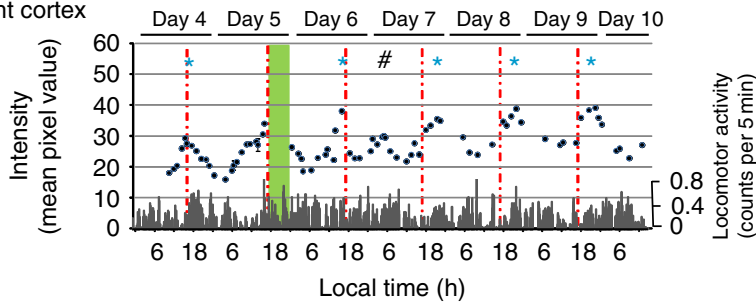

C

$\mathrm{OB}$

Local time (h)

Skin
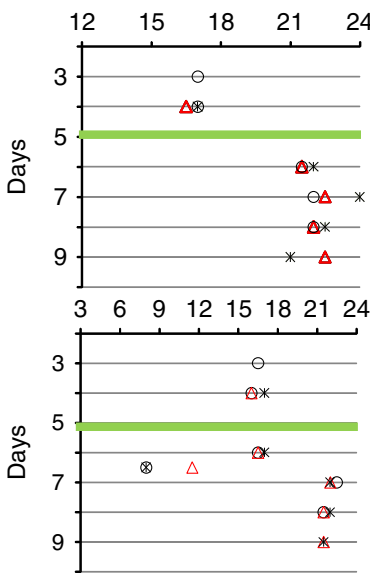

Left ear

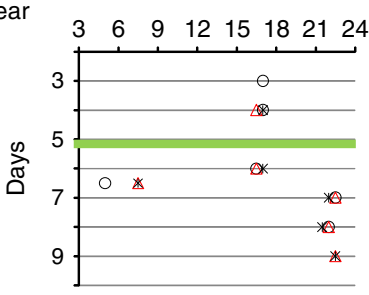

Right ear

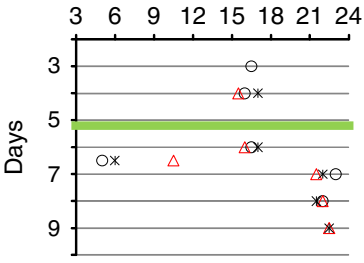

Left cortex

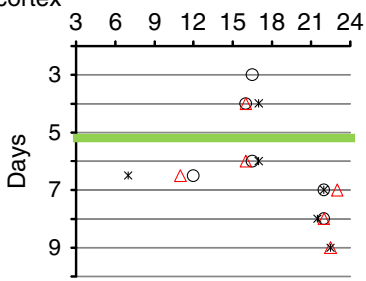

Right cortex

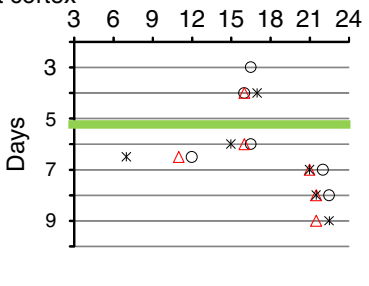

$\triangle$ Mouse1 $*$ Mouse2 $\circ$ Mouse3

Figure 6 | Different rate of photic phase-shifting process of Per1-luc expression. (a) Phase-shifting responses to light pulses in the spontaneous locomotor activity rhythms of Per1-luc mice. Activity records are expressed in black histograms of activity counts in 5 min bin and double-plotted so that $48 \mathrm{~h}$ are shown on the $x$-axis and consecutive days on the $y$-axis. Times of activity are indicated by dark regions. A yellow square shows the time zone when light pulse was exposed (CT 12-20). Red broken lines show the regression lines fitted to the onset of activity time before and after the light exposure. (b) Real-time imaging of Per1 expression in six areas of Per1-luc mice (mouse1) before and after an 8-h light pulse exposure. Green columns represent the time when light pulse were exposed. Red dash-and-dotted lines represent the peak time of Per1 expression before 8-h light pulse at day 4. *indicates the peak time of Per1-luc expression in each day. ${ }^{\# T h e ~ s e c o n d a r y ~ p e a k ~ a t ~ d a y ~} 7$ is indicated. Spontaneous locomotor activities are shown by grey histograms (counts per $5 \mathrm{~min}$ ). Luciferin was supplied by an iPRECIO pump $\left(15 \mu \mathrm{l} \mathrm{h}^{-1}\right.$ for animals). (c) Peak time in Per1 expression before and after an 8 -h light pulse exposure. The green horizontal bar shows the day (day 5) when 8-h light pulse was given to mice (mouse 1, mouse 2, mouse 3). Activity records between day 4 and day 9 were analysed for mouse 1 and mouse 2, and those between day 3 and day 8 were analysed for mouse 3 . 
at around CT 12. Previous studies using slice cultures in vitro reported Per1 gene expression rhythms that are different in different tissues ${ }^{32,33}$. The discrepancy between the present study and the previous studies is not known, but points to the possibility of effects related to the slice preparations ${ }^{34}$. For example, circadian oscillations in the peripheral tissues are reported to be affected by low temperatures ${ }^{35,36}$, slices prepared under cold conditions may change the phase of circadian oscillations. With respect to the circadian phase, our results on the first few days in DD are indeed consistent with the Per1 mRNA expression rhythms in vivo under LD condition by in situ hybridization or by quantitative polymerase chain reaction studies ${ }^{23-25}$. As is well-known, in situ hybridization and quantitative polymerase chain reaction studies require an intermittent tissue sampling at intervals of hours, which limits time resolution and includes individual differences. In this respect, the present system, tracking a single animal continuously over time, is much more powerful than the classical methods.

In addition, we succeeded in visualizing the kinetics of differential phase-shifts of circadian rhythms in six different tissues. The observation of a small secondary peak between the first and second day in five tissues suggests that a phase-delaying light pulse produced an internal transient desynchronization among the peripheral tissues, a phenomena that is commonly observed in jet-lag, shift-work-related disease, hypoinsulinemia and diabetes ${ }^{4,5}$.

In summary, we anticipate that advances using remote tracking devices, detecting low-intensity signals, eliminating noise and making improvements in automated algorithms will be forthcoming and will be widely applied to many areas of biomedical research, as well as finding applications beyond medicine. Our technology is by no means limited to circadian-related disease and research. Nevertheless, any other applications will need to investigate the sensitivity and spatial resolution if the tissue environment or source depth differs from what has been used in the present work.

\section{Methods}

Animals and housing. Mice were born and reared in the Hokkaido University animal facility where environmental conditions were controlled as follows: $12 \mathrm{~h}$ light/12 $\mathrm{h}$ dark (LD) cycle with lights on from 06:00 hours to 18:00 hours, temperature $\left(23 \pm 1{ }^{\circ} \mathrm{C}\right)$ and humidity $(50 \pm 5 \%)$. Animals were provided with food and water ad libitum. We used male adult mice (5-9-month old ) of C57BL/6J background carrying either a Per1-promoter driven firefly luciferase reporter gene (Per1-luc) or Bmal1-promoter driven enhanced beetle luciferase (ELuc) reporter gene. The Per1-luc reporter construct was as follows: a $6.7-\mathrm{kb}$ region upstream of the translation-initiation codon of $m P e r 1$ was fused to the firefly luciferase (Luc) coding region $^{37}$. The Bmall-Eluc reporter construct was as follows: an 8.1-kb region upstream of the translation-initiation codon of Bmall was fused to the ELuc coding region ${ }^{38}$. Cry $1^{-I-/ C r y 2^{-I-}}$ mice were acquired from Tohoku University $^{27}$, and bred with Perl-luc mice carrying the Per1 luciferase reporter. We back-crossed the mice for $>7$ generations and used the resulting Cry $1^{-1}-$ / Cry $2^{-1-}$-Per1-luc mice for experimentation. All animal work was performed in accordance with the Guidelines for the Care and Use of Laboratory Animals at Hokkaido University with approval (\#0800277) from the Committee for Animal Experimentation.

Ex vivo recording of bioluminescence. Per1-luc and Bmall-ELuc transgenic mice were killed by cervical dislocation and decapitated between 13:00 hours and 17:00 hours. Brains were rapidly removed and placed in ice-cold Hanks's balanced salt solution (pH 7.4, Sigma-Aldrich).

In the present study, we used horizontal OB slices because it was found in a previous study that Perl genes are highly expressed in the mitral cell layer and granular layer cells of the mouse $\mathrm{OB}^{23}$. The axons of mitral cells extend toward the frontal cortex area (horizontal direction). Furthermore, CCD camera images of the freely moving mouse OB were obtained on a horizontal plane. Therefore,

horizontal OB slices were deemed to be optimal for ex vivo Perl-luc imaging in the present study.

Slices of the whole unilateral OB slices $(300-\mu \mathrm{m}$ thick) were prepared using a microslicer (Dosaka, Osaka, Japan). OB slices at the central portion of the dorso-ventral axis were explanted on a culture membrane (Millicell CM, pore size
$0.4 \mu \mathrm{m}$, Millipore) in a 35-mm Petri dish containing DMEM (GIBCO-Invitrogen) supplemented with $\mathrm{NaHCO}_{3}(2.7 \mathrm{mM}$, Sigma-Aldrich), HEPES (10 mM, Nacalai Tesque, Japan), kanamycin (20 $\mathrm{mgl}^{-1}$, GIBCO), insulin $\left(5 \mu \mathrm{g} \mathrm{ml}^{-1}\right.$, SigmaAldrich), putrescine (100 mM, Sigma-Aldrich), human transferrin (100 $\mathrm{mg} \mathrm{m1}^{-1}$, Sigma-Aldrich), progesterone (20 nM, Sigma-Aldrich), sodium selenite (30 nM, GIBCO) and D-luciferin potassium salt $(0.1 \mathrm{mM}$, Wako Pure Chemical Industries, Japan). After sealing with Parafilm, the $\mathrm{OB}$ slices were incubated at $37^{\circ} \mathrm{C}$, and bioluminescence was monitored for $1 \mathrm{~min}$ at $10 \mathrm{~min}$ intervals with a dish type luminometer (AB2500 Kronos, ATTO, Japan). In horizontal brain slices of the whole OB, Perl and Bmall expression in the OB were measured using a PMT.

Continuous luciferin administration. Luciferin $\left(40 \mathrm{mg} \mathrm{ml}^{-1}\right)$ was intraperitoneally administered into Per1-luc mice at a controlled flow rate $\left(1 \mu \mathrm{lh}^{-1}\right.$ by an osmotic pump (Alzet, Charles River, Saint Aubin Les Elbeuf, France); 10, 15 or $30 \mu \mathrm{h} \mathrm{h}^{-1}$ by iPRECIO pump (Primtec, Tokyo, Japan)). The osmotic pump was implanted into the intraperitoneal cavity. The iPRECIO pump was connected to a 'modified free moving animal system' (complete tethering system for mice, Eicom, Shimotoba, Kyoto, Japan) which consisted of a liquid swivel (TCS1-20, 11- $\mu$ l single-channel swivel, $0.65-\mathrm{mm}$ connections), balance arm, crisscross mouse harness and a tube that was guided into the intraperitoneal cavity through a subcutaneous tunnel from an incision in the dorsal neck to a ventral abdomen incision (Supplementary Fig. 1d,e). After the operation, mice were placed in a cage until the start of the experiment.

HPLC analysis for luciferin quantification. Luciferin $\left(40 \mathrm{mg} \mathrm{ml}^{-1}\right)$ was intraperitoneally administered into Per1-luc transgenic mice at a constant flow rate by an osmotic pump (Alzet) or iPRECIO pump (Primtec). In all, 10-15 $\mu$ l of blood was collected from the tail tip and plasma was separated (Fig. 3d and Supplementary Fig. 9a). Luciferin was supplied in the morning on day 0 and sampling started at 15:00 hours on day 1 (Fig. 3d) or 11:00 hours on day 2 (Supplementary Fig. 9a). Luciferin was eluted from $5 \mu \mathrm{l}$ plasma with $95 \mu \mathrm{l}$ of ethanol containing $0.5 \%$ tri- $n$-butylphosphine at $70^{\circ} \mathrm{C}$ for $5 \mathrm{~min}$ (ref. 39). The sample was centrifuged (13,000 r.p.m., $20 \mathrm{~min}$ ) and the supernatant was filtered with a $0.45 \mu \mathrm{m}$ membrane filter (Millipore, Billerica, MA). The filtered sample was injected into a HPLC (high-performance liquid chromatography) system. Linear gradient elution (15-40\% acetonitrile/water with $0.1 \% \mathrm{TFA}, 30 \mathrm{~min}, 1.0 \mathrm{ml} \mathrm{min}^{-1}$ ) was adopted for separation with a chiral fused-silica column, CHIRAL-CEL OD-RH $(4.6 \times 150 \mathrm{~mm}$; Daicel Chemical Industry, Tokyo, Japan). Luciferin was detected with a fluorescence detector (excitation $\lambda=330 \mathrm{~nm}$, emission $\lambda=530 \mathrm{~nm}$; Supplementary Fig. 9b).

To examine the time-of-day effects on plasma luciferin levels using iPRECIO pump (10, 15 and $30 \mu \mathrm{h} \mathrm{h}^{-1}$ ), blood was collected at 4-h intervals from 11:00 hours on day 2 until 7:00 hours on day 3. Furthermore, plasma luciferin concentrations following administration at a perfusion rate of $15 \mu \mathrm{h} \mathrm{h}^{-1}$ were re-examined on days $6-$ and 7 at 4 -h intervals.

In vivo imaging system. The imaging system was set up in a custom-made imaging box (width $\times$ depth $\times$ height; $650 \times 622.5 \times 700 \mathrm{~mm}$, respectively) with a ventilating fan and sensors for controlling the temperature and humidity (Thermo Recorder TR-72 Ui, T\&D corporation, Nagano, Japan) inside a small room (width $\times$ depth $\times$ height; $2,012 \times 1,712 \times 2,200 \mathrm{~mm}$, respectively) with controlled environmental conditions (temperature $23 \pm 1^{\circ} \mathrm{C}$, humidity $50 \pm 5 \%$ ). The interior of the imaging box was coated with black materials that minimized light halation, while the front was enclosed with a black shield curtain (Supplementary Fig. 1b) Overhead lights and computer monitors were placed outside the room. The light in the room was off during imaging and the imaging box was shielded with a black curtain. In practice, the black shield curtain was not necessary for protecting penetration of lights, since the background illumination (ca. 5.0 counts per $0.5 \mathrm{~s}$ ) was not different, regardless of the curtain.

Two EM-CCD cameras (ImagEM C9100-13, effective number of pixels $512 \times 512$, cell size $16 \mu \mathrm{m} \times 16 \mu \mathrm{m}$, Hamamatsu Photonics, Hamamatsu, Shizuoka, Japan) were placed on the top panel of the imaging box at about equal angles relative to a vertical line (Supplementary Fig. 1c) using an angle adjuster (as in Fig. 1a and Supplementary Fig. 1c) in a camera box ('A' in Fig. 1a), and were fixed to the ceiling of the imaging box to observe luminescence from the animals. A cylindrical recording cage $(200 \mathrm{~mm}$ diameter, $60 \mathrm{~mm}$ in height;

Supplementary Fig. 1f) with food and a water intake nozzle connected to a water bottle (Fig. 1b and Supplementary Fig. 1f) for housing an animal for several days was placed on the central stage (as shown in Fig. 1a), which was fixed to the floor The imaging area was limited to the inner fence $(115 \mathrm{~mm} \times 115 \mathrm{~mm}, 30 \mathrm{~mm}$ in height). Two low-intensity LED light sources $(<0.01$ lux at the floor of the recording cage) illuminated the recording cage from the upper left and right (Fig. $1 \mathrm{~b}$ and Supplementary Fig. 1g) for emitting fluorescence from the scintillators (BC-490 plastic scintillator casting resin, emission $\lambda=425 \mathrm{~nm}$, Saint-Gobain Crystals, Hiram, OH, USA) that were attached to the animals (Fig. 1c and Supplementary Fig. 1e).

We did not use emission filters. The spectra of the scintillators did not practically overlap with the bioluminescence emission spectra of $L u c$ and had a limited overlapped area of $\sim 40 \mathrm{~nm}$ with that of ELuc (the wavelength of maximum 
emission; scintillators, $425 \mathrm{~nm}, L u c, 562 \mathrm{~nm}$ and $E L u c, 540 \mathrm{~nm}$ ). We examined the effect of the scintillators on the total intensity of emitted bioluminescence and found that the effect was negligible $(<0.1 \%)$. We therefore quantified the intensity of bioluminescence without emission filters in the present study.

To precisely identify the position of the luminescent target area in the recording cage, three scintillators ( 2 or $3 \mathrm{~mm}$ in diameter) were embedded around the target (ROIs) as reference markers (Supplementary Fig. 1e). Hair on the recording areas of the head and skin was shaved (Supplementary Fig. 1e). To get enough signals from freely moving mouse, the skull around the $\mathrm{OB}$ and cortex area was ground with a dental dill bur to make it thinner, which increased the intensity of bioluminescence ca. 5.3 times for the cortex and ca. 3.7 times for the OB. Finally, the recording area on the head was coated with transparent dental resin. Three scintillators were attached around the $\mathrm{OB}$ and skin (Supplementary Fig. 1e) on the Per1-luc, Bmal1-ELuc and Cry1 $1^{-1-} / \mathrm{Cry} 2^{-1-}$-Per1-luc mice.

Ultraviolet-light-emitting diode lights ( $\lambda=375 \mathrm{~nm}$, TUV550A-1, Takatsuki, Japan) were placed at the top of the recording box (Fig. 1b). The intensity of the ultraviolet-light-emitting diode lights was very low and regulated by a pulse controller (Taihou Densi, Sapporo, Hokkaido, Japan) so that ultraviolet-lightemitting diode-induced fluorescent intensity of the scintillator and bioluminescent intensity from the skin were similar with 0.5 or $1.0 \mathrm{~s}$ exposure, as recorded by the EM-CCD camera $\left(-80^{\circ} \mathrm{C}\right.$ cooling, EM-gain $=256$, max amplification $)$.

Fluorescence signals from the scintillator and bioluminescent signals from the target area were obtained using a custom video capture program (Mouse Capture). Video files were saved for subsequent analysis using the 'Mouse Tracker' program, which consists of the DuFT and SICT algorithms. Using a pattern-matching technique with model images (Fig. 2a,b and Supplementary Fig. 4), the positions of the scintillators and ROIs in the image can be automatically detected (Figs if and 2a,b; see the subsection 'Determination of ROI positions' for details). This program was made with the Matrox Imaging Library (MIL version 9.0). Circles on the image ( $\mathrm{a}$ in the Figs $1 \mathrm{f}$ and 4a,d and Supplementary Movies 2-5) indicate the location identified by the pattern-matching program in both the left and right images. The 3D position of the target can be computed only when the target area can be located in both the left and right camera images which include both true and false pattern matching (see the next section 'Automatic determination of the 3D position of scintillators' for details). Images were excluded from the analysis when the software failed to identify and trace the targets. After calibrating the bioluminescent intensity of the ROIs at each 3D position (see the subsection 'Signal-intensity calibration technique' in the 'Methods' section for details) and post-processing analysis, Perl or Bmall expression in the OB, skin, left ear, right ear, left cortex and right cortex were quantified (see the subsection 'Determination of ROI positions' for details). Pixel quantification analysis yielded gene expression data. Behavioural analysis was performed using 3D plot graphs. These analyses were performed in multiple areas over a long duration. One frame required only $0.5 \mathrm{~s}$ to obtain (except for recording the images of Bmall-ELuc mice, where $1.0 \mathrm{~s}$ was required). Luciferin ( $40 \mathrm{mg} \mathrm{ml}^{-1}$ ) was supplied by an osmotic pump or iPRECIO pump.

\section{Automatic determination of the 3D position of scintillators. The 3D}

coordinates of the scintillators on the mouse in the recording cage were derived from the 2D coordinates in a pair of images (left and right) using 'Mouse Tracker' (based on real-time tumour-tracking radiotherapy (RTRT) software) ${ }^{20,21}$. In the RTRT system, the location of a moving tumour, such as a lung cancer, can be detected every $0.03 \mathrm{~s}$ with an accuracy of $1 \mathrm{~mm}$ by tracking the position of a spherical gold surrogate marker inserted in or near the tumour using two sets of $\mathrm{X}$-ray images. The tumour is irradiated only when its location corresponds to the planned position. In this system, the tracking volume (about $<50 \mathrm{~mm}^{3}$ ) is very small and the tracing time is short. In the present study, to track gene expression in freely moving mice within the recording cage $(<200 \mathrm{~mm}$ in the $x$-, $y$-axis and $<60 \mathrm{~mm}$ in the $z$-axis) for a long duration, we replaced the gold marker and X-ray images with a scintillator and LED in DuFT for optical imaging.

The software uses a pair of transformation matrices (one each for the left and right cameras) to convert two $2 \mathrm{D}$ coordinates into a $3 \mathrm{D}$ coordinate. The matrices are used to compute a line from each camera that passes through the scintillator. The intersection point of the two lines determines the 3D coordinate. However, the two lines will often not intersect exactly due to small inaccuracies. In that case, the centre of the shortest connection between both lines is used as the scintillator position. If the length between the lines is over $1 \mathrm{~mm}$, the 3D position data are discarded during post-processing.

The transformation matrices $\mathrm{M}_{1}$ and $\mathrm{M}_{\mathrm{r}}$ are calculated as follows. Eight marker points made with a stable luminescence marker (Glowell, LUX Biotechnology), similar to the acrylic calibration cube used in the RTRT system ${ }^{20,21}$, were used to compute the transformation matrices. The points were positioned by placing a single stable luminescence marker on a rotating disc inside the recording cage (Supplementary Fig. 5a). The stable luminescence marker emits a long-lasting very stable light, generated by a chemical reaction that is produced from a gaseous tritium light source (One Glowell kit GLO-001 Green, No. X20110, Radiant flux 4, $32 \mathrm{pW}, 11.6 \times 10^{6}$ photons $/ \mathrm{s}$, luminous flux $2.14 \mathrm{nlm}$, effective wavelength $=$ $532-536 \mathrm{~nm})$. We first place the disc at the lowest height of the recording stage $(1,410 \mathrm{~mm}$ height from the bottom of recording stage as $z=0)$ and the stable luminescence marker at the maximum radius $(R=5 \mathrm{~cm})$ from the centre. We then obtain four pairs of images with the disc rotated at $45^{\circ}, 135^{\circ}, 225^{\circ}$ and $315^{\circ}$. The disc is then raised to the maximum height $(40 \mathrm{~mm} ; z=0-5)$ and four more pairs of images are obtained. We used the $2 \mathrm{D}$ coordinates of the scintillator from six of these images, along with the known 3D position of each point, to compute the transformation matrices $\left(M_{1}\right.$ and $\left.M_{r}\right)$ for each camera. $M_{l}$ and $M_{r}$ provide the equation of a straight line passing through the scintillator (Fig. 1d), that is, $[\mathrm{aM}]=[\mathrm{b}]$, where $[\mathrm{b}]$ is the $2 \mathrm{D}$ scintillator position expressed in the homogeneous coordinates (that is, the third coordinate is set to one), [a] is any position on the line (in homogeneous coordinates), and $\mathrm{M}_{1}$ and $\mathrm{M}_{\mathrm{r}}$ are $4 \times 3$ matrices of rank 3 . By measuring the image scintillator locations [a] with two EM-CCD cameras for six known stable luminescence marker positions [b], one obtains 12 equations with 12 unknowns and $\mathrm{M}_{1}$ and $\mathrm{M}_{\mathrm{r}}$ can be solved. The two matrices are stored in a file that is read by our tracking software. We also tested smaller values for the radius and height of the calibration points, but found that $\mathrm{R}=5 ; z=5$ yielded the best results.

To verify the accuracy of the $3 \mathrm{D}$ transformation matrices, additional images of the stable luminescence marker on the rotating disc were obtained every $15^{\circ}$ (24 pairs of images). The height of the disc was raised from the bottom of the cage at $10 \mathrm{~mm}$ intervals to a maximum of $40 \mathrm{~mm}$ (5 levels), and moved from the centre to a radius of $50 \mathrm{~mm}$ at $10 \mathrm{~mm}$ intervals ( 5 rings) for a total of 605 pairs of images (Supplementary Fig. 5a-c). The 2D coordinates of the stable luminescence marker on each pair of images were converted to $3 \mathrm{D}$ using the matrices computed above and compared with the actual coordinates.

We used a Kreuznach XENON (F0.95/25 mm, C-Mount; Jos. Schneider Optische, Bad Kreuznach, Germany) lens. The lens is adapted to the visible light in the range of $400-700 \mathrm{~nm}$. In this range of wavelength, the lens passes 89 and $90.0 \%$ of the bioluminescence emitted by firefly and enhanced beetle luciferase, respectively; and $82.6 \%$ of the fluorescence from the marker scintillator passes through the lens.

The lens has the focal distance around $35 \mathrm{~cm}$ (centre positon at $z=2$ ) and barrel distortion of $-2.5 \%$ at the 4 corners (Max distortion) of the square-shape image. The estimated decrease of light intensity due to the distortion was ca. $37 \%$ at the corners of the image. Actually, we found the intensity at the corner was ca. $60 \%$ of that in the centre. The differences of intensity due to distortions of the lens were compensated by the left and right cameras (Fig. 6d,e).

Signal-intensity calibration technique. Luminescence is calculated from the mean pixel value in a circular area on an image from the EM-CCD cameras. The intensity of the luminescence signal varies with the position within the recording cage, depending on the distance from the camera. To calibrate the luminescence signal, we measured the mean pixel value for the stable luminescence marker at the 605 calibration positions described in the previous section. At each calibration position, 10 images were acquired from the left and right cameras ( 10 photos at each position, total 6,050 pairs of images). The ten images were averaged to form a standard image for each camera at each position. The process was repeated three times and the results were averaged again. The mean pixel values are shown in Supplementary Fig. 5 d,e for the left $(46.19 \pm 7.65$ (mean \pm s.d.)) and right $(49.34 \pm 8.28$ (mean \pm s.d.) $)$ cameras, respectively.

The focus of the two cameras was set at a point at the centre of the cage, $z=2 \mathrm{~cm}(1,610 \mathrm{~mm}$ height from the bottom of the recording stage). The mean pixel value of the spot at this point $(x 0, y 0, z 2)$ was used as the calibration value. The ratio of the calibration value and the pixel value at each calibration point was stored in a calibration table. The calibrated pixel values are shown in Supplementary Fig. 5f. The calibrated pixel values in these figures should be constant.

The calibration points are spread on concentric rings, like the spokes of stacked wagon wheels (Supplementary Fig. 5a-c). Calibration points are spaced closer together near the centre of the cage and spread further apart near the edges. To use the calibration table for an arbitrary point in the observation cage, we first determine the eight calibration points that surround the ROI point. The distances from the ROI point to the eight calibration points are computed. The calibration values are linearly interpolated based on the computed distances. Special cases are handled when the point is near the centre $(<1 \mathrm{~cm})$, outside (in a corner of the cage) or above the calibration volume (near the ceiling).

Determination of ROI positions. A number of algorithms were developed to locate the scintillator targets on the mouse and identify the associated ROIs. All configuration parameters are specified in a Windows INI format text file. This file is used as the input to the 'Mouse Tracker' software.

In the first case, three scintillators (diameter $3 \mathrm{~mm}$ ) are placed on the mouse skin (Figs If and 2a). An image of one of the scintillators (usually about $16 \times 16$ pixels) is used as a search model for pattern matching in all frames of the video. Three instances of the model within the source target image are searched for with MIL according to specified constraints in the INI file. If three instances of the search model cannot be found in the target image, the frame is rejected and the next frame is examined. On successful identification of three scintillators, the resulting triangle is analysed to determine if the three points are actually the three scintillators and not the result of misidentification due to image noise.

The distances between the three points are computed. If the longest distance exceeds a maximum threshold specified in the INI file, the frame is rejected. Similarly, the frame is rejected if the minimum distance is less than a specified 
threshold. The distance between points depends on how the scintillators are placed on the mouse skin and the distance from the camera to the mouse when the frame was recorded. The distances can be displayed with 'Mouse Tracker' so that the user can determine reasonable values for the minimum and maximum thresholds.

The acceptance level is the value (per cent) at which the correlation (pattern match score) between the model and the pattern in the image is considered a match. A perfect match is $100 \%$ and no correlation is $0 \%$. Because the captured video images have considerable noise, perfect matches are generally unobtainable. We found that the acceptance level should be set below the default value of $70 \%$. If the acceptance level is set too high, MIL will not find any matches for the scintillators in the video frame, and if set at too low, the chance of false matches is increased and search time may also increase. The user must determine the optimal value for the acceptance level empirically, depending on the signal intensity of the target. We found that a value of about $30 \%$ worked well in our experiments (Supplementary Movies 2-5). Note that the raw video images contain both true and false pattern matching of model images.

For analysis of skin, we place the three scintillators so as to form an approximate isosceles triangle. The triangle base is assumed to be the line between the points with greatest distance. The ROI is assumed to be at the midpoint of the base line. The ROI is a circle whose diameter is set in the INI file. The ROI circle is displayed on each frame, so that the user can adjust the values for the ROI diameter. The ROI should tightly encircle the shaved region on the mouse skin where the bioluminescent signal is to be measured. We found that an ROI diameter of 12 pixels worked well. The average value of the pixels within the ROI circle is calculated and written to the output file.

For the $\mathrm{OB}$, three scintillators (diameter $2 \mathrm{~mm}$ ) are placed on the mouse head to form an approximate equilateral triangle with the $\mathrm{OB}$ at the centroid (Fig. 1f). As with skin, the diameter of the ROI circle must be empirically determined by the user, by observing the circle displayed on the video. In our experiments, an ROI diameter of 9 pixels worked well. The distances between the three scintillator points are computed. If the ROI of the $\mathrm{OB}$ cannot be identified, the frame is discarded and no further calculations are performed.

To locate other ROIs, such as the left and right ears and cortices, we developed an alternative method using a model image of the mouse head for pattern matching (Fig. $2 \mathrm{~b}$ and Supplementary Fig. 4). A representative image of the mouse head is captured and stored in a file. This image is used as a search model for pattern matching in all frames of the video with rotated versions of the model (Fig. 2b). Note that rotation of the head model significantly increases search time. More than one model head image can be specified to increase the chance of successful matches. Mouse ear ROIs (20 pixels) and cortex ROIs (8 pixels) are defined relative to the top-left corner of the model head image (Fig. 2b), specified in the INI file. If the mouse model head image is found within a video frame, the $\mathrm{OB}$ can also be located by searching for the three small scintillators on the head within the region in which the model head image was found. This decreases the chance of mismatches. Three instances of the model scintillators within the source target image are searched as described above.

If an ROI can be successfully located in both the left and right images, the 3D coordinates of the ROI can be computed. If the 3D ROI coordinates indicate an unreasonable position (that is, outside the observation cage), due to pattern mismatching, then the frame is discarded. Otherwise, the pixel values within the ROI circle of specified diameter (OB 9 pixels; Skin 12 pixels; ear 20 pixels; cortex 8 pixels) in the left and right images are averaged. The $3 \mathrm{D}$ coordinates are used to calibrate the raw pixel averages with a pre-computed calibration table. Average calibrated bioluminescent intensity from the left and right images was saved in a 'csv' file.

After analysis by 'Mouse Tracker', the data in the '.csv' file was analysed in a post-processing step. If the size of the triangle exceeds thresholds (the triangle area with three points between minimum 100-200 pixels and maximum 700-900 pixels in the OB; minimum 500-600 pixels and maximum 1,000-1,200 pixels in the skin), the frame is discarded. The distances between the three scintillator points is also checked. The remaining data was checked for the distance between the head and skin regions. If the distance exceeds a maximum threshold (default $80 \mathrm{~mm}$ ), the frame is rejected. Similarly, the frame is rejected if the distance is less than a threshold (default $20 \mathrm{~mm}$ ). Finally, gene expression in the ROI is quantified (mean pixel value) for each recording session (Supplementary Fig. 6).

Although many frames are discarded with this method, a sufficient number of good frames were found to enable the recording of the rhythmic expression of target genes (Supplementary Fig. 7a). We used the frames in which the mouse was not moving much (Supplementary Fig. 8).

The major sources of error in estimation of 3D position are twofold; the error of positioning in the measurement box and the error of pattern matching for the marker (triangle shape). On the basis of these errors, spatial resolution was calculated to be $1.63 \mathrm{~mm}$.

To calculate the 3D-positioning error, we assessed the 3D-positioning of the target area within the recording cage using the apparatus shown in Fig. 1a and based on the 605 marker points shown in Supplementary Fig. 5. From the 2D coordinates of the scintillators in each camera's image, two straight lines passing through a scintillator were calculated and the $3 \mathrm{D}$ coordinates of the scintillator were determined by calculating the intersection of these two lines (Fig. 1d). In most cases, the two lines will not intersect exactly due to small inaccuracies. We calculated the error determining intersection points by the $3 \mathrm{D}$ transformation matrices with DuFT.

Phase-shifting responses to light pulses. Perl-luc transgenic mice were maintained for at least 1 week after surgery in a LD cycle. Recording of Per1-luc expression started after transferring to DD. Mice were exposed to an 8-h light pulse at a specified CT (CT 12) on day 5 in DD. Recording was discontinued during the 8-h light exposure and resumed thereafter (Supplementary Fig. 10a). The activity onset time was designated as CT 12. For long recording, we obtained images for $10 \mathrm{~min}$, at $0.5 \mathrm{~s}$ per frame for Perl-luc within each $30 \mathrm{~min}$ interval. Locomotor activity rhythm was monitored using an on-line PC (CLOCKLAB, Actimetrics) and spontaneous locomotor activity was recorded using an infrared sensor. Daily activity onset was visually estimated from standard double-plot actograms of locomotor activity behaviour. The phase-shift in locomotor activity rhythm in response to the 8 -h light pulse was determined for several days preceding and following the day of the light pulse. A line was manually positioned through times of activity onset over several days (Fig. 6a). The primary peak time of Per1-luc expression was determined as the time of highest intensity of Per1-luc expression around a continuous high-intensity area during each circadian cycle (Fig. 6b and Supplementary Fig. 11).

Circadian rhythmicity analysis of Per1 expression in vivo. Circadian rhythmicity of quantified Perl expression was analysed using the Cosinor program (Cosiner.exe, version 2.3; Circadian Rhythm Laboratory). This program uses a least-squares regression method to fit a cosine wave to a time series and identify the best-fit cosine wave with period information. In Supplementary Table 1, a period was determined by performing cosinor analysis between $23.5 \mathrm{~h}$ and $24.0 \mathrm{~h}$ with $0.1 \mathrm{~h}$ step. This approach consists of minimizing the sum of squared deviations between the data and the fitted cosine curve. The larger this residual sum of squares is, the greater the uncertainty of the estimated parameters is. Best fitting is the percentage of variance divided by the total variance (and multiplied by 100).

The significance of the rhythm is calculated using the zero amplitude test, in which the null hypothesis is that amplitude is zero. Rejection of this null hypothesis signifies that the fitted curve (implying rhythmicity) approximates the data more closely than does a straight line of zero slope (implying constancy).

Data availability. We declare that all data supporting the findings of this study are available within the article and its Supplementary Information files.

\section{References}

1. Young, M. W. \& Kay, S. A. Time zones: a comparative genetics of circadian clocks. Nat. Rev. Genet. 2, 702-715 (2001).

2. Tei, H. et al. Circadian oscillation of a mammalian homologue of the Drosophila period gene. Nature 389, 512-516 (1997).

3. Sun, Z. S. et al. RIGUI, a putative mammalian ortholog of the Drosophila period gene. Cell 90, 1003-1011 (1997).

4. Mahoney, M. M. Shift work, jet lag, and female reproduction. Int. J. Endocrinol. 2010, 813764 (2010)

5. Marcheva, B. et al. Disruption of the clock components CLOCK and BMAL1 leads to hypoinsulinaemia and diabetes. Nature 466, 627-631 (2010).

6. Zhang, W. et al. Rapid in vivo functional analysis of transgenes in mice using whole body imaging of luciferase expression. Transgenic Res. 10, 423-434 (2001).

7. Abraham, U., Prior, J. L., Granados-Fuentes, D., Piwnica-Worms, D. R. \& Herzog, E. D. Independent circadian oscillations of Period1 in specific brain areas in vivo and in vitro. J. Neurosci. 25, 8620-8626 (2005).

8. Tahara, Y. et al. In vivo monitoring of peripheral circadian clocks in the mouse. Curr Biol. 22, 1029-1034 (2012).

9. Sakamoto, A. et al. Influence of inhalation anesthesia assessed by comprehensive gene expression profiling. Gene 356, 39-48 (2005).

10. Bellet, M. M. et al. Ketamine influences CLOCK:BMAL1 function leading to altered circadian gene expression. PLoS ONE 6, e23982 (2011).

11. Ohe, Y., Iijima, N., Kadota, K., Sakamoto, A. \& Ozawa, H. The general anesthetic sevoflurane affects the expression of clock gene mPer2 accompanying the change of NAD + level in the suprachiasmatic nucleus of mice. Neurosci Lett. 490, 231-236 (2011).

12. Kadota, K. et al. Time-dependent repression of mPer 2 expression in the suprachiasmatic nucleus by inhalation anesthesia with sevoflurane. Neurosci Lett. 528, 153-158 (2012).

13. Cheeseman, J. F. et al. Generl anesthesia alters time perception by phase shifting the circadian clock. Proc. Natl Acad. Sci. USA 109, 7061-7066 (2013).

14. Anzai, M. et al. Direct and specific effect of sevoflurane anesthesia on rat Per2 expression in the suprachiasmatic nucleus. PLOS ONE 8, e59454 (2013).

15. Mori, K. et al. Epigenetic suppression of mouse Per 2 expression in the suprachiasmatic nucleus by the inhalational anesthetic, sevoflurane. PLoS ONE 9, e87319 (2014). 
16. Saini, C. et al. Real-time recording of circadian liver gene expression in freely moving mice reveals the phase-setting behavior of hepatocyte clocks. Genes Dev. 27, 1526-1536 (2013).

17. Yamaguchi, S. et al. View of a mouse clock gene ticking. Nature 409, 684 (2001).

18. Tanaka, K. F. et al. Expanding the repertoire of optogenetically targeted cells with an enhanced gene expression system. Cell Rep. 2, 397-406 (2012).

19. Coxeter, H. S. M. Projective Geometry 2nd edn (Springer-Verlag, 1987).

20. Shirato, H., Shimizu, S., Shimizu, T., Nishioka, T. \& Miyasaka, K. Real-time tumour-tracking radiotherapy. Lancet 353, 1331-1332 (1999).

21. Shirato, H. et al. Physical aspects of a real-time tumor-tracking system for gated radiotherapy. Int. J. Radiat. Oncol. Biol. Phys. 48, 1187-1195 (2000).

22. Otsu, N. A threshold selection method from gray-level histograms. IEEE Trans. Syst. Man. Cybernet 9, 62-66 (1979).

23. Hamada, T., Honma, S. \& Honma, K. Light responsiveness of clock genes, Per1 and Per2, in the olfactory bulb of mice. Biochem. Biophys. Res. Commun. 409, 727-731 (2011).

24. Tanioka, M. et al. Molecular clocks in mouse skin. J. Invest. Dermatol. 129, 1225-1231 (2009).

25. Wakamatsu, H. et al. Restricted-feeding-induced anticipatory activity rhythm is associated with a phase-shift of the expression of mPer1 and mPer2 mRNA in the cerebral cortex and hippocampus but not in the suprachiasmatic nucleus of mice. Eur. J. Neurosci. 13, 1190-1196 (2001).

26. Nelson, W., Tong, Y. L., Lee, J. K. \& Halberg, F. Methods for cosinorrhythmometry. Chronobiologia 6, 305-323 (1979).

27. van der Horst, G. T. et al. Mammalian Cry1 and Cry2 are essential for maintenance of circadian rhythms. Nature 398, 627-630 (1999).

28. Beersma et al. The progression of circadian phase during light exposure in animals and humans. J. Biol. Rhythms 24, 153-160 (2009).

29. de Chaumont, F. et al. Computerized video analysis of social interactions in mice. Nat. Methods 9, 410-417 (2012)

30. Pérez-Escudero, A., Vicente-Page, J., Hinz, R. C., Arganda, S. \& de Polavieja, G. G. idTracker: tracking individuals in a group by automatic identification of unmarked animals. Nat. Methods 11, 743-748 (2014)

31. Yoshikawa, T. et al. Spatiotemporal profiles of arginine vasopressin transcription in cultured suprachiasmatic nucleus. Eur. J. Neurosci. 42, 2678-2689 (2015).

32. Abe, M. et al. Circadian rhythms in isolated brain regions. J. Neurosci. 22 , 350-356 (2002).

33. Yamazaki, S. et al. Resetting central and peripheral circadian oscillators in transgenic rats. Science 288, 682-685 (2000).

34. Okada, M., Sakaguchi, T. \& Kawasak,i, K. Correlation between anti-ubiquitin immunoreactivity and region-specific neuronal death in N-methyl-D-aspartatetreated rat hippocampal organotypic cultures. Neurosci Res. 22, 359-366 (1995).

35. Morf, J. et al. Cold-inducible RNA-binding protein modulates circadian gene expression posttranscriptionally. Science 338, 379-383 (2012).

36. Buhr, E. D., Yoo, S. H. \& Takahashi, J. S. Temperature as a universal resetting cue for mammalian circadian oscillators. Science 330, 379-385 (2010)

37. Inagaki, N., Honma, S., Ono, D., Tanahashi, Y. \& Honma, K. Separate oscillating cell groups in mouse suprachiasmatic nucleus couple photoperiodically to the onset and end of daily activity. Proc. Natl Acad. Sci. USA 104, 7664-7669 (2007).
38. Nakajima, Y. et al. Enhanced beetle luciferase for high-resolution bioluminescence imaging. PLoS ONE 5, e10011 (2010).

39. Niwa, K., Nakamura, M. \& Ohmiya, Y. Stereoisomeric bio-inversion key to biosynthesis of firefly D-luciferin. FEBS Lett. 580, 5283-5287 (2006).

\section{Acknowledgements}

This research was supported by Creation of Innovation Centers for Advanced Interdisciplinary Research Areas Program, Ministry of Education, Culture, Sports, Science and Technology, Japan; in part by JSPS KAKENHI (grant number 24621001); by a research fund from The Suhara Memorial Foundation; a research fund from Research Foundation for Opto-Science and Technology; and was partially supported by Special Expenditures of 'Reverse Translational Research from Advanced Medical Technology to Advanced Life Science; from Real-time Tracking Technology to Real-time Tracking Life Science' funded by the Japanese Ministry of Education, Culture, Sports, Science and Technology. We are grateful to Dr Y. Ohmiya and Dr Y. Nakajima for providing us with the Bmall-Eluc transgenic mice and Dr K. Niwa for their technical advice of measuring the luciferin concentration in the blood. We thank Dr Michael C. Antle for his comments on the drafts of this manuscript.

\section{Author contributions}

T.H., K.H. and S.H. conceived, and T.H. designed overall experiments. T.H., M.I and H.S. conceived the DuFT system, and T.H. and M.I. produced the DuFT. T.H., M.I., K.S. and N.M. made the algorithm of the DuFT. N.M. made the program controlling two EM-CCD cameras. K.S., N.M. and H.S. made the program of determination of 3D position, ROI position, and calibration of luminescence. T.H., M.I., K.S. and N.M. performed the inspection of accuracy of DuFT apparatus using control light. T.H. and K.S. performed the inspection of accuracy of quantification of bioluminescence in freely moving mice. T.H. conceived, produced the recording cage for long recording for in vivo imaging and performed mouse experiments. T.H. and K.S. analysed the data of experiments using mouse. T.H., M.I., H.S., S.H. and K.H. contributed regents/materials/analysis tools. T.H., K.S., S.H., and K.H. wrote the paper, all authors reviewed the manuscript.

\section{Additional information}

Supplementary Information accompanies this paper at http://www.nature.com/ naturecommunications

Competing financial interests: The authors declare no competing financial interests.

Reprints and permission information is available online at http://npg.nature.com/ reprintsandpermissions/

How to cite this article: Hamada, T. et al. In vivo imaging of clock gene expression in multiple tissues of freely moving mice. Nat. Commun. 7:11705 doi: 10.1038/ncomms11705 (2016).

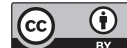

This work is licensed under a Creative Commons Attribution 4.0 International License. The images or other third party material in this article are included in the article's Creative Commons license, unless indicated otherwise in the credit line; if the material is not included under the Creative Commons license, users will need to obtain permission from the license holder to reproduce the material. To view a copy of this license, visit http://creativecommons.org/licenses/by/4.0/ 